\title{
The Electronic Structure and Stability of Germanium Tubes $\mathrm{Ge}_{30} \mathrm{H}_{12}$ and $\mathrm{Ge}_{33} \mathrm{H}_{12}$
}

\section{Long Van Duong, ${ }^{a}$ Eduard Matito ${ }^{\text {b,c }}$, Miquel Solà, ${ }^{\text {d,* }}$ Hadi Behzadi, e,* Mohammad Jafar Momeni ${ }^{\mathrm{e}}$ and Minh Tho Nguyen ${ }^{\text {a,f,** }}$}

${ }^{a}$ Institute for Computational Science and Technology (ICST), Quang Trung Software City, Ho Chi Minh City, Vietnam

${ }^{b}$ Donostia International Physics Center (DIPC), Donostia, Euskadi, Spain

${ }^{c}$ Ikerbasque Foundation for Science, 48011 Bilbao, Euskadi, Spain

${ }^{d}$ Institut de Química Computacional i Catàlisi (IQCC) and Departament de Química, Universitat de Girona, C/ Maria Aurèlia Capmany, 69, 17003 Girona, Spain

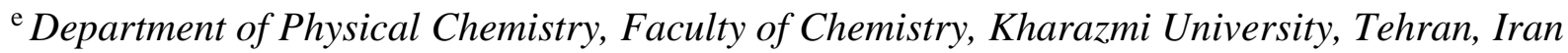

${ }^{\mathrm{f}}$ Department of Chemistry, KU Leuven, Celestijnenlaan 200F, B-3001 Leuven, Belgium 


\begin{abstract}
Geometries of non-tetrahedral and ultrastable silicon and germanium nanocrystals $\mathrm{X}_{18} \mathrm{H}_{12}$ and $\mathrm{X}_{19} \mathrm{H}_{12}(\mathrm{X}=\mathrm{Si}, \mathrm{Ge})$ have recently been predicted for the development of cluster-based nanomaterials for energy and microengineering purposes. To further explore the possibility of larger Ge clusters, we investigated in this work the molecular and electronic structure of the germanium tube $\mathrm{Ge}_{30} \mathrm{H}_{12}$, composed of six parallel, planar hexagons using DFT calculations. Insertion of $\mathrm{Ge}$ atoms at the center of three inner hexagons of $\mathrm{Ge}_{30} \mathrm{H}_{12}$ leads to a $\mathrm{Ge}_{33} \mathrm{H}_{12}$ tube, which is also an energy minimum structure. The electronic structure and molecular orbital shapes of these tubes can be predicted by the wavefunctions of a particle on a hollow cylinder model and a cylinder model. Different aromaticity indices including PDI, $\mathrm{I}_{\mathrm{ring}}, \mathrm{I}_{\mathrm{NG}}, \mathrm{MCI}$, and $\mathrm{I}_{\mathrm{NB}}$, as well as the electron localization function (ELF) were calculated to evaluate the electron delocalization and the aromaticity of the Ge tubes considered.
\end{abstract}




\section{Introduction}

Since the discovery of benzene in 1825 by Faraday, aromaticity was introduced as the key concept in the realm of organic chemistry to describe the stability, molecular structure and reactivity of many organic molecules. ${ }^{1-4}$ Due to the persistent ambiguity in the definition of aromaticity and its non-observable nature, it has been a subject of continuing debate among chemists. Despite the controversy in the definition of aromaticity, aromaticity still remains as a useful, even fundamental, concept for the interpretation of structural and chemical properties of a variety of classes of organic molecules. ${ }^{5}$ Moreover, the discovery of aromatic inorganic/organometallic clusters promises to expand its scope of application. ${ }^{6-11}$ Many indices have been proposed in the literature for direct and quantitative measurement of aromaticity, which can be categorized into four main groups, including energy, structure, electron delocalization, and magnetic based indices. ${ }^{12,13}$ Accordingly, it has been recommended that a set of indices, rather than a sole index, should employed for characterization of aromatic compounds. ${ }^{14}$ The final results would be more reliable if different aromaticity indices provided consistent results for a set of compounds. The necessity of different aromaticity indices has been discussed in several recent reviews. ${ }^{1,5,15-18}$

Triggered by benzene discovery, chemists have long been seeking to expand the concept of aromaticity to inorganic molecules, especially to analogous silicon compounds ${ }^{19,20}$ Regarding this interest, many attempts were made to synthesize and predict stable aromatic silicones. For example, Scheschkewitz et al. synthesized a silicon counterpart to benzene, with bulky organic substituents instead of hydrogen, showing high stability based on electron delocalization and aromaticity. $^{21}$ 
Silicon and germanium nanocrystals and nanostructures have drawn the attention of researchers for their potential application in energy conversion, energy storage, light-emitting diode, and memory devices. Nowadays solar cells based on silicon nanocrystals find their way into the energy production market and germanium nanostructures are being studied as high-capacity anode materials for Li-ion batteries. ${ }^{22-26}$

Recently, $\operatorname{Vach}^{27}$ predicted the aromaticity of the electron-deficient tubular $\mathrm{Si}_{19} \mathrm{H}_{12}$. The predicted $\mathrm{Si}_{19} \mathrm{H}_{12}$ contains three parallel and planar hexagons and one additional $\mathrm{Si}$ atom located in the middle hexagon. The central $\mathrm{Si}$ atom is multicoordinated and $\mathrm{Si}-\mathrm{Si}$ bonds are characterized by electron deficient properties. Electron deficiency keeps electrons delocalized through the whole structure, and ultimately provides aromatic character to the system. Vach's calculations showed that $\mathrm{Si}_{19} \mathrm{H}_{12}$ is thermodynamically more stable than $\mathrm{Si}_{18} \mathrm{H}_{12}$ and such a stability is related to a strong electron delocalization. ${ }^{27}$ Using electronic and magnetic criteria, it was shown that the overcoordinated $\mathrm{Si}_{19} \mathrm{H}_{12}$ has electron deficient bonds and is more aromatic than benzene. Due to electron-deficient aromaticity, electronic and optical properties of $\mathrm{Si}_{19} \mathrm{H}_{12}$ are totally different from the $\mathrm{Si}_{18} \mathrm{H}_{12}$ nanotube, ${ }^{28}$ which may result in light-harvesting applications such as solar cells and optoelectronic devices. ${ }^{29}$ It was also shown that $\mathrm{Ge}_{18} \mathrm{H}_{12}$ nanocrystals, similar to $\mathrm{Si}_{18} \mathrm{H}_{12}$, could be further stabilized by insertion of one germanium atom into the center of the middle hexagon, and exhibit some electron-deficient aromaticity. Our previous results showed that the electrondeficient aromaticity concept could be extended to Ge based materials. ${ }^{30}$ The presence of such aromaticity is intriguing and has stimulated us to further explore the possibility of larger Ge clusters being stabilized by this bonding phenomenon. ${ }^{31}$

It is likely that the feature observed in tubular silicon and germanium species arises from a type of aromaticity that is called tubular or cylindrical aromaticity, as recently reported by some 
of authors of the present study. ${ }^{32,33}$ This aromaticity is explained by the hollow cylinder model (HCM) in which the Schrödinger equation can be solved for a particle moving in a hollow cylindrical box. ${ }^{34}$ Accordingly, the shape of the eigenstates obtained from the HCM is quasiidentical with that of molecular orbitals calculated from quantum chemical methods.

In this context, we set out to examine the electronic structures of two extended tubular germanium tubes including $\mathrm{Ge}_{30} \mathrm{H}_{12}$ and $\mathrm{Ge}_{33} \mathrm{H}_{12}$ making use of the hollow cylinder model (HCM) and cylinder model (CM), respectively. Also, different electronic and magnetic indices are analyzed to further shed light on the aromaticity of these compounds. The proposed germanium tubes in this study and their silicon counterparts are expected to play a role in the following generation of energy storage and energy conversion devices.

\section{Computational Methods}

Standard electronic structure calculations and geometry optimizations are performed within the framework of density functional theory (DFT) using hybrid B3LYP functional and the 6$31 \mathrm{G}(\mathrm{d})$ basis set ${ }^{35-37}$ with the aid of the Gaussian 09 program. ${ }^{38}$ Harmonic vibrational frequency calculations at the same level reveal only real vibrational frequencies and thus reported structures are minima of the potential energy surface. As for aromaticity indices, several criteria based on electron delocalization measures are employed. ${ }^{12,13,39}$ These indices measure the cyclic electron delocalization of mobile electrons in aromatic rings. First, we study the para-delocalization index $(\mathrm{PDI})^{17,35}$ which is obtained using the delocalization index (DI $)^{41,42}$ as defined in the framework of the QTAIM of Bader. ${ }^{43-45}$ The PDI is an average of all DI of para-related atoms in a given six- 
membered ring. For mono-determinantal closed-shell wavefunctions, the DI between atoms $A$ and $B$ is given by:

$$
\begin{gathered}
\delta(A, B)=4 \sum_{i, j}^{o c c . M O} S_{i j}(A) S_{i j}(B) \\
\delta(A, B)=4 \sum_{i, j}^{o c c . M O} S_{i j}(A) S_{i j}(B)
\end{gathered}
$$

The summations in Eq. (1) run over all occupied molecular orbitals (MOs). $\mathrm{S}_{i j}(A)$ is the overlap between MOs $i$ and $j$ within the basin of atom $A . \delta(A, B)$ provides a quantitative indication on the number of electrons delocalized or shared between atoms $A$ and $B$. If the atoms are defined in the Hilbert space, the latter quantity is known as the Mayer bond order. ${ }^{46}$ We also employ a set of four multicenter indices, namely, the $I_{\text {ring }}, I_{N G}, \mathrm{MCI}$, and $I_{N B}$. For a ring structure represented by the $A=\left[A_{1}, A_{2}, \ldots A_{\mathrm{n}}\right]$ string, the multicenter index $\left(I_{\text {ring }}\right)$ of Giambiagi et al. ${ }^{15}$ for a closed-shell monodeterminantal wavefunction is defined as follows:

$$
\begin{gathered}
I_{\text {ring }}(A)=2^{N} \sum_{i_{1}, i_{2} \cdots \cdots i_{N}}^{o c c . M O} S_{i_{1} i_{2}}\left(A_{1}\right) S_{i_{2} i_{3}}\left(A_{2}\right) \cdots S_{i_{N} i_{1}}\left(A_{N}\right) \\
I_{\text {ring }}(\mathcal{A})=2^{N} \sum_{i_{1}, i_{2}, \cdots, i_{N}}^{o \alpha c . M O} S_{i_{1} i_{2}}\left(A_{1}\right) S_{i_{2} i_{3}}\left(A_{2}\right) \cdots S_{i_{N} i_{1}}\left(A_{N}\right)
\end{gathered}
$$

Some of us proposed a normalized version of the $I_{\text {ring }}$ index, ${ }^{47} I_{N G}$, which is expected to be less dependent on the ring size than its unnormalized analogs, and for aromatic species is given by:

$I_{N G}(A)=\frac{\pi^{2}}{4 N N_{\pi}} I_{\text {ring }}{ }^{1 / N}$ 


$$
I_{N G}(\mathcal{A})=\frac{\pi^{2}}{4 N N_{\pi}} I_{\text {ring }}{ }^{1 / N}
$$

where $N$ is the total number of atoms in the ring and $N_{\pi}$ the total number of $\pi$ electrons. $I_{N G}$ has the peculiarity of reproducing the so-called TREPE ${ }^{48}$ values at the Hückel MO theory. ${ }^{49}$

According to Bultinck and coworkers ${ }^{50}$, summing up all the $I_{\text {ring }}$ values resulting from the permutations of indices $A_{1}, A_{2}, \ldots A_{N}$ defines a new index of aromaticity, the multicenter index (MCI) whose formula reads:

$\operatorname{MCI}(A)=\frac{1}{2 N} \sum_{P(A)} I_{\text {ring }}(A)$

$$
\operatorname{MCI}(\mathcal{A})=\frac{1}{2 N} \sum_{P(\mathcal{A})} I_{\text {ring }}(\mathcal{A})
$$

where $P(\mathrm{~A})$ stands for a permutation operator acting over string $A$ interchanging the atomic labels $A_{1}, A_{2}, \ldots, A_{N}$ to generate up to the $N$ ! permutations of the elements in the string. Generally, the values of MCI and $I_{\text {ring }}$ are in tight correlation because the dominant contribution to $\mathrm{MCI}$ is the Kekulé structure, nonetheless some exceptions may arise. ${ }^{47}$ Finally, there is a normalized version of the MCI index for aromatic rings ${ }^{47}, I_{N B}$, given by:

$$
\begin{aligned}
I_{N B}(A) & =\frac{C}{N N_{\pi}}[2 N \cdot M C I(A)]^{1 / N} \\
I_{N B}(\mathcal{A}) & =\frac{C}{N N_{\pi}}[2 N \cdot M C I(\mathcal{A})]^{1 / N}
\end{aligned}
$$

where $C \approx 1.5155$. For the series of indices defined above, the higher the PDI, $I_{\text {ring }}, I_{N G}, \mathrm{MCI}$, and $I_{N B}$ values, the more aromatic a six-membered ring is. The expressions $I_{N B}$ and $I_{N G}$ used in this work do not include the term $G\left(N_{\pi}\right)$ which was given in the original article ${ }^{47}$ It is worth mentioning 
that none of the multicenter indices aforedescribed can be easily computed for rings of more than twelve members. ${ }^{51}$

Calculation of atomic overlap matrices $(\mathrm{AOM})$ and computations of the DI, $I_{\text {ring }}, I_{N G}, \mathrm{MCI}$, and $I_{N B}$ are performed with the AIMPAC ${ }^{52}$ and ESI-3D ${ }^{53-55}$ collection of programs. Calculations of the DIs with DFT cannot be performed exactly because the electron-pair density is not available at this level of theory. ${ }^{56}$ As an approximation, we use the Kohn-Sham orbitals obtained from a DFT calculation to compute Hartree-Fock-like DIs through Eq. (1) and, therefore, we do not expect to recover electron correlation effects.

We also analyze the electron localization function (ELF) using the TopMod program. ${ }^{57}$ As shown by Savin et al., ${ }^{58}$ the ELF measures the excess of kinetic energy density due to the Pauli repulsion. In the region of space where the Pauli repulsion is strong the ELF is close to one, whereas where the probability of finding same-spin electrons close together is high, the ELF tends to zero. For an $N$-electron single determinantal closed-shell wavefunction built from Hartree-Fock (HF) or Kohn-Sham orbitals, the ELF is given by ${ }^{59,60}$ :

$$
\begin{gathered}
\eta=\frac{1}{1+\left(\frac{D}{D_{h}}\right)^{2}}, \\
\eta=\frac{1}{1+\left(\frac{\mathrm{D}}{\mathrm{D}_{\mathrm{h}}}\right)^{2}},
\end{gathered}
$$

Where

$$
D=\frac{1}{\rho} \nabla_{2}^{2} \Gamma^{(2) \sigma \sigma}\left(\overrightarrow{r_{1}}, \overrightarrow{r_{2}}\right)=\frac{1}{2} \sum_{j=1}^{N}\left|\nabla \phi_{j}\right|^{2}-\frac{1}{8} \frac{|\nabla \rho|^{2}}{\rho}
$$




$$
\begin{gathered}
\frac{D=\frac{1}{\rho} \nabla_{2}^{2} \Gamma^{(2) \sigma \sigma}\left(\vec{r}_{1}, \vec{r}_{2}\right)=\frac{1}{2} \sum_{j=1}^{N}\left|\nabla \phi_{j}\right|^{2}-\frac{1}{8} \frac{|\nabla \rho|^{2}}{\rho}}{D_{h}=\frac{3}{10}\left(3 \pi^{2}\right)^{2 / 3} \rho^{5 / 3}} \\
\quad \underline{\mathrm{D}_{\mathrm{h}}=\frac{3}{10}\left(3 \pi^{2}\right)^{2 / 3} \rho^{5 / 3}} \\
\rho=\sum_{j=1}^{N}\left|\phi_{j}\right|^{2}, \\
-\rho=\sum_{\mathrm{j}=1}^{\mathrm{N}}\left|\phi_{\mathrm{j}}\right|^{2}
\end{gathered}
$$

and where $N$ is the number of electrons and $\Gamma^{(2) \sigma \sigma}\left(\vec{r}_{1}, \vec{r}_{2}\right)$ is the same-spin contributions to the pair density. Since the ELF is a scalar function, an analysis of its gradient field can be carried out to locate its attractors (local maxima) and the corresponding basins. There are basically two chemical types of basins: the core basins (C) and the valence (V) ones, which are characterized by their synaptic order, i.e., the number of core basins with which they share a common boundary. ${ }^{61}$ Graphical representations of the bonding are obtained by plotting isosurfaces of the ELF. These isosurfaces delimit volumes within which the Pauli repulsion is rather weak. The localization domains are called irreducible when they contain only one attractor, and reducible otherwise. The reduction of reducible domains is another criterion of discrimination between basins, and the reductions occur at a critical value of the bonding isosurface. The domains are ordered with respect to the ELF critical values, yielding bifurcations (tree diagrams). The ELF bifurcation values can be also taken as a measure of aromaticity. According to Santos and co-workers ${ }^{62}$, aromatic 
compounds are characterized by high bifurcation values and small differences in bifurcation values of different basins.

The electronic structure of $\mathrm{Ge}_{30} \mathrm{H}_{12}$ is also examined by the hollow cylinder model (HCM $)^{32}$, ${ }^{33}$ while the electronic structure of $\mathrm{Ge}_{33} \mathrm{H}_{12}$ is examined by the cylinder model (CM). ${ }^{63}$ The latter can be considered as a special case of the former when the inner radius of the hollow cylinder is neglected. The Schrödinger equation for a particle moving in a hollow cylinder was solved by Gravesen and co-workers ${ }^{34}$ and later on by Miliodoros for a cylinder with Möbius topology. ${ }^{64}$ The key point, which causes a difference between the two models is related to the different boundary conditions, i.e., the CM is a special case of the HCM when the inner radius $\left(\mathrm{R}_{0}\right)$ is neglected. A change in the boundary conditions leads to a change in the Schrödinger equation solution for $P(\rho)$ function among the separable solution $\psi(\rho, \Theta, z)=P(\rho) \Theta(\theta) Z(z)$. This leads to a change in the ordering of eigenstates (wavefunctions), such that the cylinder model becomes more suitable for a narrow nanotube-like molecular structure. ${ }^{63}$

In general, the energy spectrum in both HCM and CM is quantized with three quantum numbers, namely, the rational $k(k=1,2,3, \ldots)$, the rotational $l(l=0, \pm 1, \pm 2, \pm 3, \ldots)$, and the radial $n(n=$ $1,2,3, \ldots)$, and it is defined by the expressions:

$$
\begin{aligned}
& E=\frac{\hbar^{2}}{2 m R^{2}}\left(\left(k_{l n} R\right)^{2}+\left(\frac{k \pi}{L^{f}}\right)^{2}\right) \\
& E=\frac{\hbar^{2}}{2 m R^{2}}\left(\left(\beta_{l n} R\right)^{2}+\left(\frac{k \pi}{L^{f}}\right)^{2}\right)
\end{aligned}
$$

for HCM (eq.10) and CM (eq.11), respectively. In Eqs. (10) and (11), $m$ is the mass of the particle moving in the cylinder, $R$ is the radius of the cylinder, $L^{f}=L / R$ with $L$ being the length of the cylinder, and $k_{l n} R$ is obtained from boundary conditions. 


\section{Results and Discussion}

The optimized structures of $\mathrm{Ge}_{30} \mathrm{H}_{12}$ and $\mathrm{Ge}_{33} \mathrm{H}_{12}$ and the nomenclature used for $\mathrm{Ge}$ atoms are shown in Figure 1. The tube $\mathrm{Ge}_{30} \mathrm{H}_{12}$ consists of five $\mathrm{Ge}_{6}$ rings containing $12 \mathrm{GeA}, 12 \mathrm{GeB}$, and 6 $\mathrm{GeC}$ atoms, piled on top of each other. The tube $\mathrm{Ge}_{33} \mathrm{H}_{12}$ is also comprised of two $\mathrm{Ge}_{6}$ rings (12 $\mathrm{GeA})$ at the outermost and three innermost $\mathrm{Ge}_{6}$ units $(12 \mathrm{GeB}, 6 \mathrm{GeC}, 2 \mathrm{GeE}$, and one $\mathrm{GeD}$ atoms). In hosting three additional Ge atoms along the symmetry axis, the tubular $\mathrm{Ge}_{30} \mathrm{H}_{12}$ maintains a prism identity. The inserted $\mathrm{GeE}$ atom is positioned in the center of the structure, and 2 of $\mathrm{GeD}$ atoms are deviated from the center of the six neighbor Ge atoms by about $0.2 \AA$. By introducing Ge atoms inside the $\mathrm{Ge}_{30} \mathrm{H}_{12}$ cage, the $\mathrm{Ge}-\mathrm{Ge}$ bonds in three innermost and two outermost units are elongated by about 0.2 and $0.1 \AA$, respectively. No significant changes in the angles are found upon insertion of Ge atoms, except for the $\mathrm{H}-\mathrm{GeA}-\mathrm{GeB}$ angle values that change from $130^{\circ}$ in $\mathrm{Ge}_{30} \mathrm{H}_{12}$ to $123^{\circ}$ in $\mathrm{Ge}_{33} \mathrm{H}_{12}$.

Our previous results pointed out that after introduction of one $\mathrm{Ge}$ atom into $\mathrm{Ge}_{18} \mathrm{H}_{12}$, the resulting $\mathrm{Ge}_{19} \mathrm{H}_{12}$ structure stabilizes $4.5 \mathrm{eV}$ with respect to the isolated units. ${ }^{30} \mathrm{We}$ attribute this stabilization to the increased aromatic character, as proved by different analysis. In this work, upon insertion of three $\mathrm{Ge}$ atoms into the $\mathrm{Ge}_{30} \mathrm{H}_{12}$ tube, $\mathrm{Ge}_{33} \mathrm{H}_{12}$ stabilizes $21.0 \mathrm{eV}$ (see Figure 1). In the two following subsections, we analyze in detail the electronic structure of both tubular forms.

\section{$3.1 \mathrm{Ge}_{30} \mathrm{H}_{12}$}

The tubular $\mathrm{Ge}_{30} \mathrm{H}_{12}$ includes $54 \mathrm{Ge}-\mathrm{Ge}$ and $12 \mathrm{Ge}-\mathrm{H}$ bonds and it is-also contains 132 valence electrons. According to the Lewis theory, this molecule fits perfectly with a structure of 132 valence electrons organized in 66 2c-2e bonds. However, the natural geometry of the tubular 
moiety tends to create some delocalized electrons within the structure and decrease the number of localized electrons in the structure.

Figure 2.a displays the atom-in-molecule (AIM) topology maps, including 54 Ge-Ge and 12 Ge-H bond critical points (BCP, in green), 29 ring critical points (RCP, yellow points), and 4 cage critical bonds (CCP, in blue). While the Laplacian $\nabla^{2} \rho_{\boldsymbol{r}}$ at $54 \mathrm{Ge}-\mathrm{Ge}$ BCPs is negative, for the 12 Ge-H BCPs is positive, suggesting that the 54 Ge-Ge bonds behave as classical covalent bonds, and the $12 \mathrm{Ge}-\mathrm{H}$ are closed-shell bonds. ${ }^{65,} 66$ The electron densities at Ge-H BCPs are also remarkable larger than those at Ge-Ge BCPs, suggesting a great "donor-acceptor" character of the Ge-H bonds.

The $\nabla^{2} \rho_{r}$ Laplacian contour maps in different planes of $\mathrm{Ge}_{30} \mathrm{H}_{12}$ are shown in Figure 3.a to 3.e. Figure 3.a displays great charge concentration regions belonging to the hydrogen atoms. Figure 3.c - 3.e show that the GeA-GeA, GeB-GeB, and GeC-GeC bonds display a similar electronic distribution, while Figures $3 . a-3 b$ show small charge concentration in GeB-GeC bonds. Although the Laplacian $\nabla^{2} \rho_{r}$ at the 54 Ge-Ge BCPs is negative, the Laplacian values at $12 \mathrm{GeB}$ $\mathrm{GeC}$ BCPs are close to zero, suggesting less localized electrons in $\mathrm{GeB}-\mathrm{GeC}$ bonds than in the other Ge-Ge bonds. The Mayer bond orders ${ }^{46,67}$ of $\mathrm{Ge}_{30} \mathrm{H}_{12}$ given in Table 1 also show larger values for the $\mathrm{Ge}-\mathrm{H}$ bonds than for the $\mathrm{GeB}-\mathrm{GeC}$ bonds. In order to understand these properties, we now examine the electronic structure of the tubular $\mathrm{Ge}_{30} \mathrm{H}_{12}$ by analyzing the interaction between the MOs of $\mathrm{Ge}_{30}$ skeleton and the MOs of $2\left[\mathrm{H}_{6}\right]$ units $\left(2 \mathrm{x}\left[\mathrm{H}_{6}\right]\right)$.

The electron structure of small nanotubes such as the series boron double ring $B_{2 n}(n=10$ 14), ${ }^{33}$ the triple ring tubular $\mathrm{B}_{27}{ }^{+},{ }^{32}$ and the skeleton $\mathrm{Si}_{15}$ in the triple ring $\mathrm{Mn}_{2} @ \mathrm{Si}_{15}{ }^{68}$ have been investigated using the hollow cylinder model $(\mathrm{HCM}) .{ }^{63}$ In these anti-prism structures, valence MOs can be separated in three different sets: 2c-2e MOs (s-MOs), radial MOs (r-MOs) and 
tangential (t-MOs). Recently, the HCM has been applied to a prism structure, the skeleton $\mathrm{Si}_{12}$ of $\mathrm{Cr} @ \mathrm{Si}_{12}$, whose whole valence MOs fit in a unique HCM. ${ }^{63}$ The fact that only one HCM is required for all valence MOs is due to the $\mathrm{sp}^{3}$ hybridization of silicon atoms. However, the latter destabilizes the $\mathrm{Si}_{12}$ skeleton.

Germanium is isovalent with silicon, and the tubular skeleton $\mathrm{Ge}_{30}$ consists of five $\mathrm{Ge}_{6}$ rings. Therefore, we also find the whole set of valence MOs of the tubular skeleton $\mathrm{Ge}_{30}$ obey only one HCM with the parameters $\varepsilon=0.77$ and $L^{f}=5.85$. The radius and the height of the skeleton $\mathrm{Ge}_{30}$ amount to $9.87 \AA$ and $2.44 \AA$, respectively. The active radius $r(r=\varepsilon . R)$ of $1.88 \AA$, which is deduced from the HCM, is shorter as compared to the value of $2.11 \AA$ of the Ge van der Waals radius. ${ }^{69}$ The height of the hollow cylinder model $L\left(L=L^{f} * R\right)$ is $14.26 \AA$. Compared to the real height of $9.86 \AA$ of the skeleton $\mathrm{Ge}_{30}$ (i.e., the distance between the top and bottom rings of the skeleton), the height of the hollow cylinder in $\mathrm{Ge}_{30}$, in which electrons move, is larger by $\Delta L=$ $2.20 \AA(\Delta L=(14.26-9.86) / 2)$. The active radius of $\mathrm{HCM}$ for the skeleton $\mathrm{Ge}_{30}$ of $\mathrm{Ge}_{30} \mathrm{H}_{12}$, and that of $\mathrm{HCM}$ applied to the skeleton $\mathrm{Si}_{12}$ of $\mathrm{Cr} @ \mathrm{Si}_{12}$ are not significantly different from each other, while the extended length in $\mathrm{Ge}_{30}$ is obviously much longer than that in $\mathrm{Si}_{12}\left(\mathrm{Ge}_{30}\right.$ includes 5 rings while $\mathrm{Si}_{12}$ has only 2 rings).

As previously indicated for the $\mathrm{Si}_{12}$ skeleton, ${ }^{70}$ the different behavior in the three sets s-MOs, r-MOs, and t-MOs and the interaction with atomic orbitals of $\mathrm{Cr}$ lead to a separation of these sets in the silicon double ring. Therefore, the different behavior in three sets s-MOs, r-MOs, and t-MOs of the skeleton $\mathrm{Ge}_{30}$ becomes obvious. However, interaction between the MOs of $\mathrm{Ge}_{30}$ skeleton and the MOs of $2 \times\left[\mathrm{H}_{6}\right]$ will result in a transfer of delocalized electrons above and below the $\mathrm{Ge}_{30}$ tube to the localized $\mathrm{Ge}-\mathrm{H}$ bonds in $\mathrm{Ge}_{30} \mathrm{H}_{12}$. 
Thus, we can only consider the two classical sets: the $\pi$-MOs set from r-MOs and the $\sigma$-MOs set from s-MOs and t-MOs. In $\mathrm{Ge}_{30} \mathrm{H}_{12}$, we have 6 MOs in $\pi$-MOs set and 60 MOs in $\sigma$-MOs set. Figure 5 shows the formation of the set of $\pi$-MOs of $\mathrm{Ge}_{30} \mathrm{H}_{12}$ by contributions of MOs from $2 \mathrm{x}$ $\left[\mathrm{H}_{6}\right]$ into the $\pi$-MOs set of the $\mathrm{Ge}_{30}$ skeleton. It is easily recognized that all these MOs are $\mathrm{Ge}-\mathrm{H}$ anti-bonding orbitals. With $20 \pi$-electrons, the $\mathrm{Ge}_{30}$ skeleton follows the $4 \mathrm{~N}+2 \mathrm{M}$ rule with $\mathrm{N}=3$ and $M=4, N$ and $M$ being the number of degenerated and non-degenerated $\pi$-MOs, respectively. Let us define the difference between MOs energies as:

$$
\Delta \mathrm{MO}=E_{\mathrm{MO}(\mathrm{Ge} 30 \mathrm{H} 12)}-E_{\mathrm{MO}(\mathrm{Ge} 30)}
$$

where $E_{\mathrm{MO}(\mathrm{Ge} 30 \mathrm{H} 12)}$ and $E_{\mathrm{MO}(\mathrm{Ge} 30)}$ are the energies of the MOs in $\mathrm{Ge}_{30} \mathrm{H}_{12}$ and $\mathrm{Ge}_{30}$, respectively, which are assigned the same quantum number from the HCM. All $\triangle \mathrm{MO}$ values are positive for the $\pi$-MOs interaction.

In both ( $\mathrm{k} 02$ )-orbital and $(\mathrm{k} \pm 12)$-orbital, the $\Delta \mathrm{MO}$ values increase along with the rational quantum number $\mathrm{k}$. Therefore, in $\mathrm{Ge}_{30}$, the occupied MOs with high $\mathrm{k}$ rapidly become the unoccupied MOs in $\mathrm{Ge}_{30} \mathrm{H}_{12}$. As a result, $10 \pi$-MOs in $\mathrm{Ge}_{30}$ are formed while the $\pi$-MOs set of $\mathrm{Ge}_{30} \mathrm{H}_{12}$ has now $6 \mathrm{MOs}$. Hence, reduction of delocalized radial electrons is a way to stabilize the tubular structure.

In $\mathrm{Ge}_{30} \mathrm{H}_{12}$, there are $27 \sigma-\mathrm{MOs}$ with negative $\Delta \mathrm{MO}$ values. The $15 \mathrm{MOs}$ having the largest $|\Delta \mathrm{MO}|$ values are shown in Figure 6 . These tangential orbitals follow the $4 \mathrm{~N}+2 \mathrm{M}$ rule with $\mathrm{N}=$ 4 and $\mathrm{M}=7$. We can easily recognize that the large $\mathrm{H}$ contributions correspond to interactions between $\mathrm{H}$ orbitals and the $\mathrm{p}_{\mathrm{z}}$-orbitals of the GeA moieties, whereas the small $\mathrm{H}$ contributions correspond to interactions between $\mathrm{H}$ orbitals and s-orbitals of $\mathrm{Ge}$ atoms. 
The results obtained for different indices of aromaticity are given in Table 2 . In order to provide some reference values, let us mention that PDI, $\mathrm{I}_{\mathrm{ring}}, \mathrm{I}_{\mathrm{NG}}, \mathrm{MCI}$, and $\mathrm{I}_{\mathrm{NB}}$ for benzene at the B3LYP/6-311G++(d,p) level are 0.103 e, 0.048 e, 0.041 e, 0.072 e, and 0.041 e, respectively. ${ }^{5}$ The comparison of these values with those of Table 2, reveals that the aromaticity of the $\mathrm{Ge}_{6}$ rings is not particularly large. All indices considered indicate that the aromaticity follows the order $\mathrm{Ge}_{6}(\mathrm{~B})$ $>\mathrm{Ge}_{6}(\mathrm{C})>\mathrm{Ge}_{6}(\mathrm{~A})$. The aromatic character of the most aromatic $\mathrm{Ge}_{6}(\mathrm{~B})$ ring within $\mathrm{Ge}_{30} \mathrm{H}_{12}$ ranges from about $50 \%$ of that of benzene according to $\mathrm{I}_{\mathrm{NG}}$ and $\mathrm{I}_{\mathrm{NB}}$ indices to less than the $10 \%$ given by $\mathrm{I}_{\text {ring }}$ and MCI. Not unexpectedly, given the approximate $\mathrm{sp}^{3}$ hybridization of its Ge atoms, the least aromatic ring is the outer one.

To further analyze the aromaticity of $\mathrm{Ge}_{30} \mathrm{H}_{12}$ we have also performed an ELF bifurcation analysis of this system (see Figure 7). We find that the separation of the six tangential Ge-Ge bond basins of the inner $\mathrm{Ge}_{6}$ rings $\left(\mathrm{Ge}_{6}(\mathrm{~B}) \& \mathrm{Ge}_{6}(\mathrm{C})\right)$ occurs at a value $\mathrm{ELF}=0.48$. At $\mathrm{ELF}=0.49$, the $\mathrm{Ge}-\mathrm{Ge}$ tangential bonding basins of the three inner $\mathrm{Ge}_{6}$ rings are separated from the radial valence Ge basins. At ELF $=0.51$, separation of the Ge-Ge tangential bonding basins of the outer-most $\mathrm{Ge}_{6}$ ring (2 rings) takes place. Finally, at $\mathrm{ELF}=0.58$, separation of the radial valence Ge-Ge basins into two parts occurs and, at ELF $=0.60$, a complete separation of radial basins is observed. According to these results, two conclusions can be drawn: i) First, the radial aromaticity $(\mathrm{BV}(\mathrm{ELF})=0.60)$ is higher than the tangential one $(\mathrm{BV}(\mathrm{ELF})=0.51)$, and ii) the tangential aromaticity of the different $\mathrm{Ge}_{6}$ rings is quite similar (BV(ELF) ranging from 0.48 to 0.51 ).

\section{$3.2 \mathrm{Ge}_{33} \mathrm{H}_{12}$}

Figure 2.b shows the AIM maps of the $\mathrm{Ge}_{33} \mathrm{H}_{12}$ electron density, including $74 \mathrm{Ge}-\mathrm{Ge}$ and 12 Ge-H BCPs (in green), 56 RCPs (yellow points), and 14 CCPs (in blue). 
Although the Laplacian $\nabla^{2} \rho_{\boldsymbol{r}}$ values at the GeA-GeA, GeA-GeB, and GeA-H BCPs (which are masked by presence of $\mathrm{GeE}$ and $\mathrm{GeD}$ atoms) are not remarkably changed with respect to those of $\mathrm{Ge}_{30} \mathrm{H}_{12}$, the Laplacian $\nabla^{2} \rho_{\boldsymbol{r}}$ at the GeB-GeB, GeC-GeC, and GeB-GeC BCPs presents positive values, therefore, $\mathrm{GeB}-\mathrm{GeB}, \mathrm{GeC}-\mathrm{GeC}$, and $\mathrm{GeB}-\mathrm{GeC}$ bonds become more closed-shell-like bonds.

The $\nabla^{2} \rho_{\boldsymbol{r}}$ Laplacian contour maps in different planes of $\mathrm{Ge}_{33} \mathrm{H}_{12}$ are shown in Figures 3.f to 3.j. The latter Figures show a large charge concentration area in GeA-GeA, GeA-GeB, GeA-H, and GeE-GeD bonds. While Figures 3.f - 3g indicate no charge concentration between the GeB-GeC bonds, Figures 3.i - 3.j display a reduced charge concentration area between the GeB-GeB and GeC-GeC bonds. Finally, Figure 3.f points out a large charge concentration area within the GeEGeD bonds.

The presence of three Ge atoms inside the $\mathrm{Ge}_{33} \mathrm{H}_{12}$ tube permits the circulation of electrons through the cylinder more freely than in the hollow $\mathrm{Ge}_{30} \mathrm{H}_{12}$. In our previous study, ${ }^{63}$ the ground state valence $\mathrm{MO}$ of the triple ring $\mathrm{B}_{27}{ }^{+}$and the fullerene-like $\mathrm{B}_{14}$ have been used as a standard model to decide whether the hollow cylinder model or the cylinder model should be applied. Figure 4 displays a ground valence MO, $\mathrm{HOMO}-40$ of the $\mathrm{Ge}_{30}$ skeleton (Figure 4.b) and a ground valence MO, HOMO - 45, of the $\mathrm{Ge}_{33}$ skeleton (Figure 4.d). The shape of the HOMO - 40 of the $\mathrm{Ge}_{30}$ skeleton suggests that the hollow cylinder model is suitable for the $\mathrm{Ge}_{30}$ skeleton, whereas the shape of $\mathrm{HOMO}-45$ of the $\mathrm{Ge}_{33}$ skeleton indicates that the cylinder model should be used for the latter compound.

Figure 8 shows a few MOs from the $\mathrm{Ge}_{33}$ skeleton and the corresponding ones obtained from the cylinder model, proving the excellent predictability of the cylinder model for this kind of geometrical structures. In particular the shape of the $\left(\begin{array}{lll}1 & 0 & 3\end{array}\right)$ eigenstate, which corresponds to the 
$\mathrm{HOMO}-2$ of the $\mathrm{Ge}_{33}$ skeleton, it is not only reproduced but its relative energy is also correctly predicted by the cylinder model.

The parameters of the cylinder model derived for the $\mathrm{Ge}_{33}$ skeleton are shown in Figure 4.c. The height of the cylinder model amounts to $12.34 \AA$, while the height of the $\mathrm{Ge}_{33}$ skeleton is 10.30 $\AA$. Accordingly, the height of the current cylinder model is extended by a small length of $\Delta \mathrm{L}=$ $1.02 \AA$ in each direction. The active radius of the cylinder model is $4.16 \AA$, while the average radius of the $\mathrm{Ge}_{33}$ skeleton (from five rings) is $2.52 \AA$. This means that the electrons within the $\mathrm{Ge}_{33}$ skeleton can move in a cylinder whose radius is larger than the radius of the structure by $\Delta \mathrm{R}$ $=1.62 \AA$.

Although the $\mathrm{Ge}_{33}$ skeleton is actually larger than the $\mathrm{Ge}_{30}$ counterpart, the height of the cylinder in which the electrons of $\mathrm{Ge}_{33}$ are moving becomes smaller than the height of the hollow cylinder in which electrons of $\mathrm{Ge}_{30}$ circulate. The extended height of the cylinder of $\mathrm{Ge}_{33}$ is also smaller than the extended height of the hollow cylinder of $\mathrm{Ge}_{30}$. The shape of the ground state valence MOs can partly be predicted considering this phenomenon.

According to the $\mathrm{HCM}$, the electrons in the $\mathrm{Ge}_{30}$ can move in and out of the tubular structure to distances up to $1.88 \AA$ whereas, in $\mathrm{Ge}_{33}$, the $\mathrm{CM}$ predicts that the electrons can move in and out of the tubular structure up to $1.62 \AA$.

In general, the presence of three central Ge atoms vertically placed inside the tubular structure reduces the available volume for moving electrons. As it has three more Ge atoms, more valence electrons are added to the system, and the tubular $\mathrm{Ge}_{30}$ also donates electrons to these central atoms. The net charges given in Table 3 show that large negative charges are located on GeE and GeD positions. Moreover, Table 1 displays that the largest Mayer bond orders correspond 
to the GeE-GeD bonds, while the Mayer bond orders of GeB-GeB, GeB-GeC, GeC-GeC, GeB$\mathrm{GeD}$, and $\mathrm{GeC}-\mathrm{GeE}$ bonds are significantly smaller.

Figure 9 shows the formation of the set of $\pi$-MOs of $\mathrm{Ge}_{33} \mathrm{H}_{12}$ from contributions of MOs from $2 \times\left[\mathrm{H}_{6}\right]$ into the $\pi$-MOs set of $\mathrm{Ge}_{33}$ skeleton. At the first glance, the ground state $\pi$-MOs set is not formed by the $p_{\mathrm{xy}}$ hybrid, but rather by the $s$ hybrid. However, formation of the (k 0 2)orbitals still leads to anti-bonding orbitals / interactions between the $\mathrm{Ge}_{33}$ skeleton and hydrogen atoms $2 \times\left[\mathrm{H}_{6}\right]$. The (4 0 2)-orbital and (5 0 2)-orbital are occupied MOs in the $\mathrm{Ge}_{33}$ skeleton, whereas, in $\mathrm{Ge}_{33} \mathrm{H}_{12}$, these orbitals are unoccupied.

The $p_{\mathrm{xy}}$ orbitals have larger contributions to the $(\mathrm{k} \pm 12)$-orbitals. The $\left(\begin{array}{l}4 \pm 1 \\ 2\end{array}\right)$-orbitals are strong bonding MOs with an energy difference of $2.24 \mathrm{eV}$ between the HOMO - 13, HOMO - 13' of $\mathrm{Ge}_{33} \mathrm{H}_{12}$ and $\mathrm{HOMO}-3, \mathrm{HOMO}-3^{\prime}$ of $\mathrm{Ge}_{33}$ skeleton. The $\left(5 \pm 1\right.$ 2)-eigenstates of $\mathrm{Ge}_{33} \mathrm{H}_{12}$ cylinder are assigned to the HOMO - 2 and HOMO - 3' with an energy of $0.38 \mathrm{eV}$ lower than the HOMO and HOMO' of the $\mathrm{Ge}_{33}$ skeleton.

Figure 10 indicates formation of some $\sigma$-MOs with large $\Delta \mathrm{MO}$ values. Bonding MOs are found for the ( $\mathrm{k} 3$ 1)-eigenstates, and $(\mathrm{k} \pm 1$ 1)-eigenstates with large $\mathrm{k}$ values. As a result, the delocalized basins located above and below the $\mathrm{Ge}_{33}$ skeleton become localized basins in the Ge-H bonds.

$\mathrm{Ge}_{33} \mathrm{H}_{12}$ consists of five $\mathrm{Ge}_{6}$ rings piled on top of each other. The outermost rings $1-\mathrm{Ge}_{6}$ and $5-\mathrm{Ge}_{6}(\mathrm{GeA}$ atoms in Figure $1 \mathrm{~b})$, which are equivalent by symmetry, and the three innermost ones can also be considered as three $\mathrm{Ge}_{7}$ units ( $\mathrm{GeB}$ and $\mathrm{GeC}$ atoms Figure 1b). Results in Table 4 show that the aromatic character of $\mathrm{Ge}_{6}$ rings is small for the outer rings, but substantial for the inner rings. All indices indicate that the aromaticity follows the ordering $\mathrm{Ge}_{6}(\mathrm{~B})>\mathrm{Ge}_{6}(\mathrm{C})>\mathrm{Ge}_{6}(\mathrm{~A})$. The aromatic character of the most aromatic $\mathrm{Ge}_{6}(\mathrm{~B})$ ring in $\mathrm{Ge}_{33} \mathrm{H}_{12}$ ranges from about 50\% (PDI, 
$\mathrm{I}_{\mathrm{NG}}$, and $\mathrm{I}_{\mathrm{NB}}$ ) to less than the $5 \%$ given by $\mathrm{I}_{\text {ring }}$ and MCI. Overall, the outer rings of $\mathrm{Ge}_{33} \mathrm{H}_{12}$, as those of $\mathrm{Ge}_{30} \mathrm{H}_{12}$, have a low (if any) aromatic character. Conversely, the inner rings of $\mathrm{Ge}_{33} \mathrm{H}_{12}$ have a significant aromatic character.

The ELF plots of $\mathrm{Ge}_{33} \mathrm{H}_{12}$ (Figure 11) show that a separation of the six tangential Ge-Ge bonding basins of the three inner $\mathrm{Ge}_{6}$ rings $\left(\mathrm{Ge}_{6}(\mathrm{~B}) \& \mathrm{Ge}_{6}(\mathrm{C})\right)$ occurs at $\mathrm{ELF}=0.48$. At this ELF value, there are Ge-Ge radial bonding basins between the three Ge atoms in the center of the cylinder. At ELF $=0.51$, a separation of the Ge-Ge tangential bonding basins of the outermost $\mathrm{Ge}_{6}$ ring ( 2 rings) is observed. Then, at ELF $=0.58$, the Ge-Ge radial bonding basins of the three innermost $\mathrm{Ge}_{6}$ units are separated from each other. At $\mathrm{ELF}=0.60$, the Ge valence basins in the tangential $\mathrm{Ge}_{6}(\mathrm{C})$ unit are separated from the radial Ge-Ge bonding basins, and, finally, at ELF = 0.62, the radial Ge-Ge bonding basins are completely separated from each other. From these ELF results, three conclusions emerge: i) First, the radial aromaticity $(B V(E L F)=0.62)$ is larger than the tangential one $(\mathrm{BV}(\mathrm{ELF})=0.51)$, and ii) the tangential aromaticity of different $\mathrm{Ge}_{6}$ rings is quite similar to each other (BV(ELF) ranging from 0.48 to 0.51$), 3$ ) suggesting a similar aromatic character of both clusters $\mathrm{Ge}_{30} \mathrm{H}_{12}$ and $\mathrm{Ge}_{33} \mathrm{H}_{12}$.

\section{Concluding Remarks}

This paper investigates the geometries, chemical bonding, MOs analysis, and aromaticity of two hydrogenated germanium tubes $\mathrm{Ge}_{30} \mathrm{H}_{12}$ and $\mathrm{Ge}_{33} \mathrm{H}_{12}$. We have shown that the MOs of $\mathrm{Ge}_{30} \mathrm{H}_{12}$, obtained from DFT calculations, are effectively reproduced by the interaction between the MOs of $\mathrm{Ge}_{30}$ skeleton, predicted by HCM, and the MOs of 12 hydrogen atoms. Also, the MOs of the $\mathrm{Ge}_{33} \mathrm{H}_{12}$ structure can basically be reproduced using the cylinder model, considering $\mathrm{Ge}_{33} \mathrm{H}_{12}$ 
as resulting from interaction of a $\mathrm{Ge}_{33}$ tubular skeleton and 12 hydrogen atoms. The aromaticity analysis of $\mathrm{Ge}_{30} \mathrm{H}_{12}$ and $\mathrm{Ge}_{33} \mathrm{H}_{12}$ suggests that both structures have similar aromatic character. In the two clusters, the inner rings show more aromatic character that the outer rings. Moreover, the radial aromaticity is found to be larger than the tangential counterpart. The results obtained showing the critical role of cylindrical aromaticity in the stability of these clusters could be important in potential emerging technologies.

\section{Acknowledgements}

H.Behzadi and M.J. Momeni gratefully acknowledge the financial support of Kharazmi University (Gran Number: D/2059). LVD and MTN thank the Department of Science and Technology of Ho Chi Minh City, Vietnam, for support under the Grant 196/2016/HĐ-SKHCN. This work has been also supported by the Ministerio de Economía y Competitividad (MINECO) of Spain (Projects CTQ2017-85341-P to M.S. and CTQ2014-52525-P and EUIN2017-88605 to E.M., the Basque Government (Project IT588-13), and the Generalitat de Catalunya (Project 2017SGR39 and ICREA Academia 2014 prize for M.S.). E.M. also acknowledges financial support from Ikerbasque Foundation.

\section{Authors Information}

- Long Van Duong, Email: longphysics06@gmail.com, ORCID: 0000-0003-3349-3035

- Miquel Solà, Email: miquel.sola@udg.edu, ORCID: 0000-0002-1917-7450

- Eduard Matito, Email: ematito@gmail.com, ORCID:0000-0001-6895-4562

- Hadi Behzadi, Email: behzadi@khu.ac.ir , ORCID: 0000-0003-4070-5809 
- Jafar Momeni, Email: jafar.momeni@gmail.com,

- Minh Tho Nguyen, Email: minh.nguyen@kuleuven.be, ORCID: 000-0002-3803-0569 
Table 1. Mayer bond orders of $\mathrm{Ge}_{30} \mathrm{H}_{12}$ and $\mathrm{Ge}_{33} \mathrm{H}_{12}$.

\begin{tabular}{|c|c|c|c|c|c|c|c|c|c|}
\hline Cluster & $\mathbf{G e}_{\mathrm{A}}-\mathrm{H}$ & $\mathbf{G e}_{\mathrm{A}}-\mathbf{G e}_{\mathrm{A}}$ & $\mathbf{G e}_{\mathrm{A}}-\mathbf{G e}_{\mathbf{B}}$ & Geв-Geв & Geв-Gec & Gec-Gec & GeB-GeD & Gec-GeE & GeE-Ged \\
\hline $\mathrm{Ge}_{30} \mathrm{H}_{12}$ & 0.886 & 0.872 & 0.760 & 0.801 & 0.688 & 0.743 & - & - & - \\
\hline $\mathrm{Ge}_{33} \mathrm{H}_{12}$ & 0.892 & 0.855 & 0.734 & 0.554 & 0.486 & 0.567 & 0.508 & 0.436 & 1.087 \\
\hline
\end{tabular}


Table 2. PDI (electrons), $I_{\text {ring }}$ (electrons), $I_{N G}$, MCI (electrons), and $I_{N B}$ results for the $\mathrm{Ge}_{30} \mathrm{H}_{12}$ at the B3LYP/6-31g(d) level.

\begin{tabular}{|l|l|l|l|}
\hline Aromaticity index & Ge6(A) & $\mathbf{G e}_{6}(\mathbf{B})$ & $\mathbf{G e}_{6}(\mathbf{C})$ \\
\hline PDI & 0.0066 & 0.0197 & 0.0134 \\
\hline I $_{\text {ring }}$ & 0.0004 & 0.0018 & 0.0010 \\
\hline $\mathrm{I}_{\mathrm{NG}}$ & 0.0183 & 0.0240 & 0.0216 \\
\hline $\mathrm{MCI}$ & 0.0006 & 0.0026 & 0.0017 \\
\hline $\mathrm{I}_{\mathrm{NB}}$ & 0.0187 & 0.0237 & 0.0220 \\
\hline
\end{tabular}


Table 3. The net charges (in electrons) of $\mathrm{H}$ and $\mathrm{Ge}$ atoms of $\mathrm{Ge}_{30} \mathrm{H}_{12}$.

\begin{tabular}{llllll}
\hline Cluster & $q_{H}$ & $q_{G e A}$ & $q_{G e B}$ & $q_{G e C}$ & $q_{G e \text {-ins }}$ \\
\hline Ge30H12 & -0.080 & 0.135 & -0.084 & 0.056 & \\
& & & & & GeD: -0.332 \\
Ge33H12 & -0.089 & 0.125 & 0.017 & 0.104 & GeE: -0.592 \\
\hline
\end{tabular}


Table 4. PDI (electrons), $I_{\text {ring }}$ (electrons), $I_{N G}$, MCI (electrons), and $I_{N B}$ results for the $\mathrm{Ge}_{33} \mathrm{H}_{12}$ at the B3LYP/6-31g(d) level. ${ }^{\mathrm{a}}$

\begin{tabular}{|l|l|l|l|}
\hline Aromaticity index & $\mathbf{G e}(\mathbf{A})$ & $\mathbf{G e}(\mathbf{B})$ & $\mathbf{G e}(\mathbf{C})$ \\
\hline PDI & 0.0053 & 0.0491 & 0.0329 \\
\hline $\mathrm{I}_{\text {ring }}$ & 0.0003 & 0.0009 & 0.0007 \\
\hline $\mathrm{I}_{\mathrm{NG}}$ & 0.0173 & 0.0213 & 0.0203 \\
\hline $\mathrm{MCI}$ & 0.0005 & 0.0027 & 0.0042 \\
\hline $\mathrm{I}_{\mathrm{NB}}$ & 0.0181 & 0.0237 & 0.0255 \\
\hline
\end{tabular}

${ }^{a} \mathrm{MCI}$ values of the seven-membered rings of $\mathrm{Ge}$ give $\mathrm{MCI}=-0.0011$ for $\mathrm{Ge}_{7}(\mathrm{~B})$ and $\mathrm{MCI}=0.0019$ for 3$\mathrm{Ge}_{7}(\mathrm{C})$. 


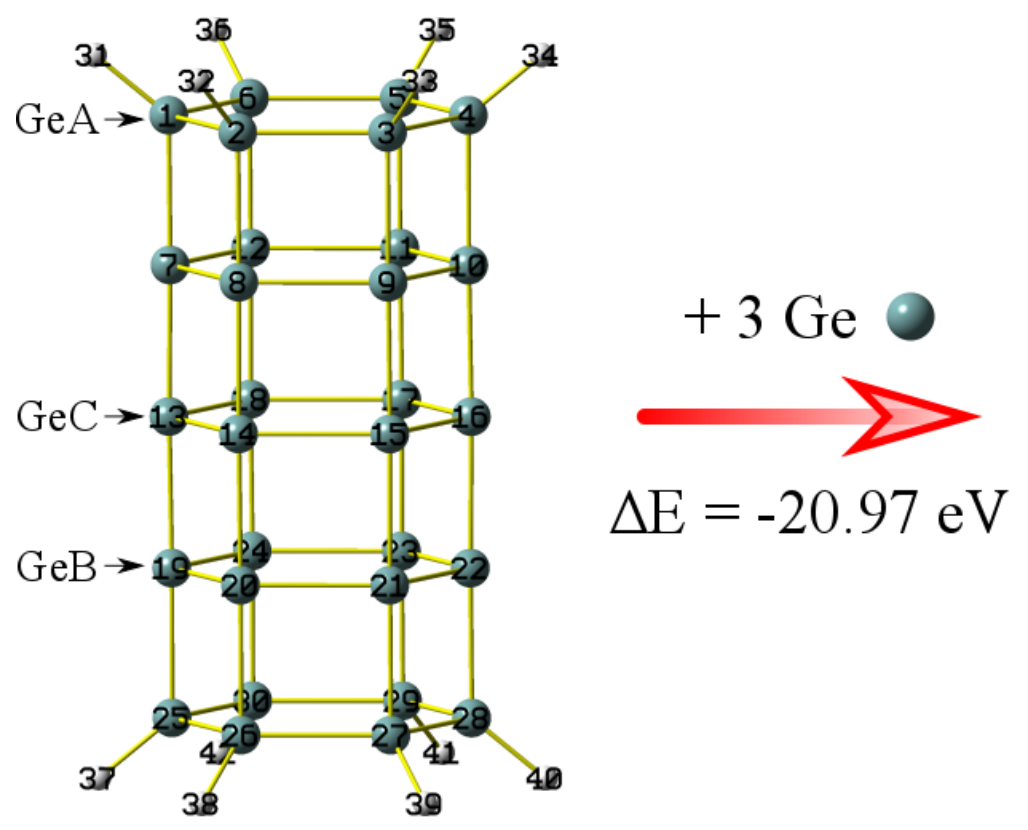

a)

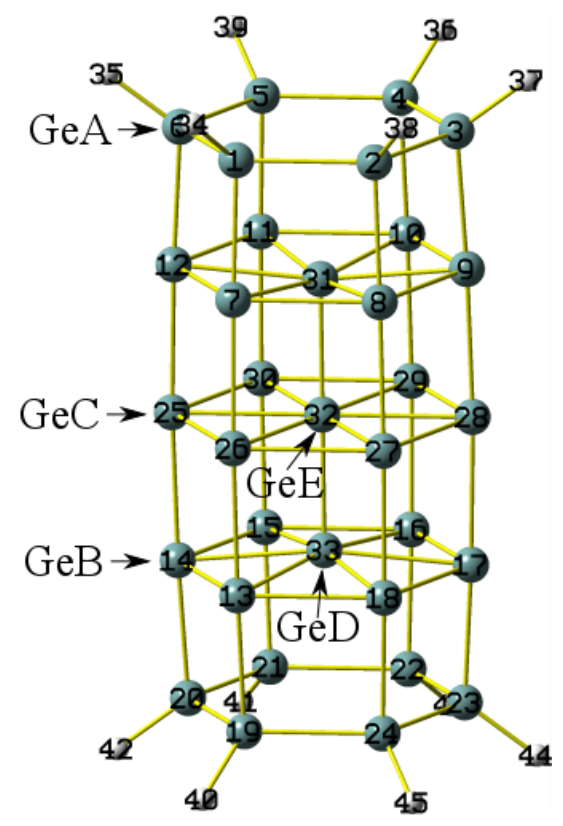

b)

Figure 1. Optimized structures of a) $\mathrm{Ge}_{30} \mathrm{H}_{12}$ and b) $\mathrm{Ge}_{33} \mathrm{H}_{12} \mathrm{NCs}$ along with the nomenclature used for Ge atoms. 


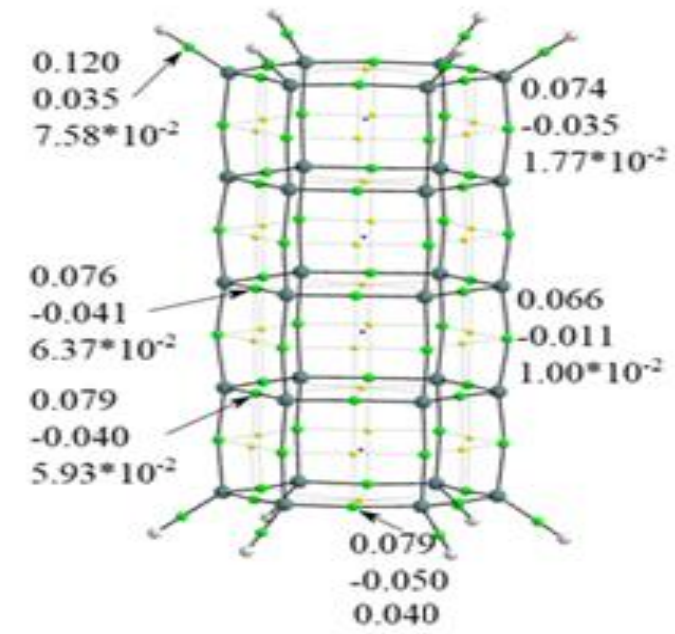

a)

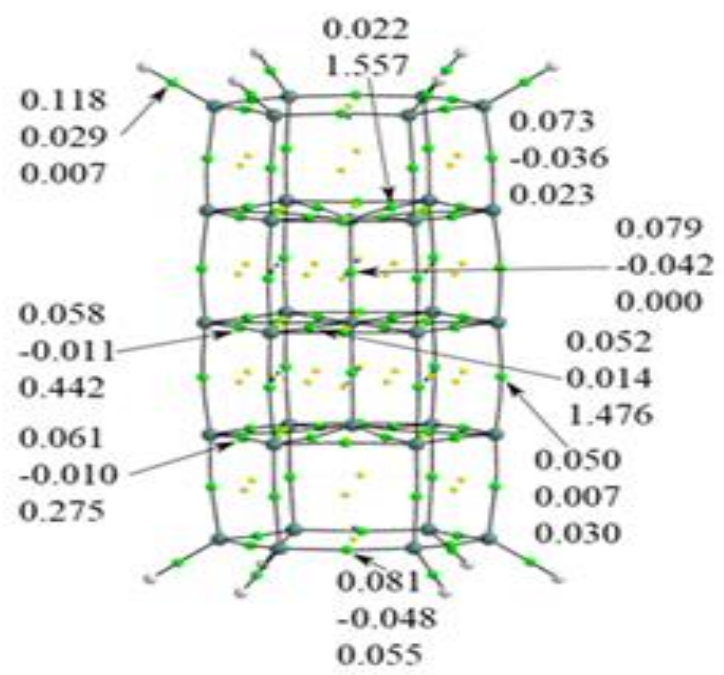

b)

Figure 2. a) The AIM graphs of $\mathrm{Ge}_{30} \mathrm{H}_{12}$ including $54 \mathrm{Ge}-\mathrm{Ge}$ and $12 \mathrm{Ge}-\mathrm{H}$ bond critical points (green points), 29 ring critical points (yellow points), and 4 cage critical bonds (blue points). b) The AIM graphs of $\mathrm{Ge}_{33} \mathrm{H}_{12}$ including $74 \mathrm{Ge}-\mathrm{Ge}$ and $12 \mathrm{Ge}-\mathrm{H}$ bond critical points (green points), 56 ring critical points (yellow points), and 14 cage critical bonds (blue points). The value of electron density, Laplacian, and bond ellipticity at bond critical points are shown in three entries, one on top of each other. 


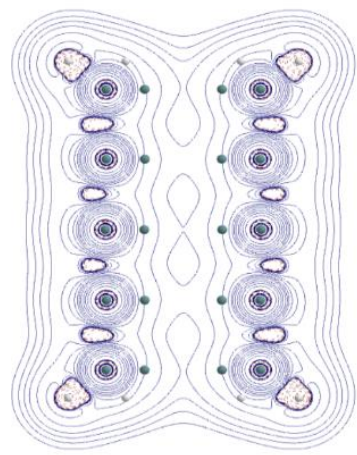

a)

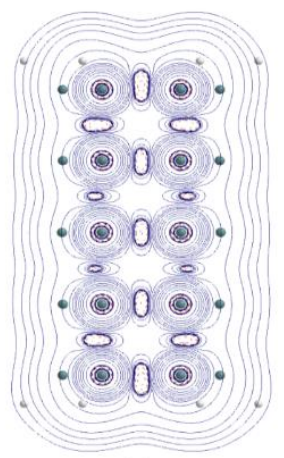

b)

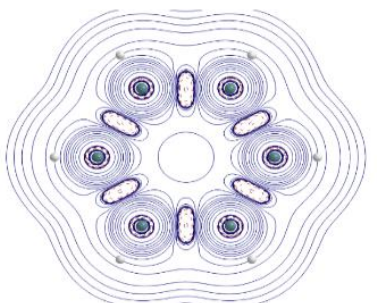

c)

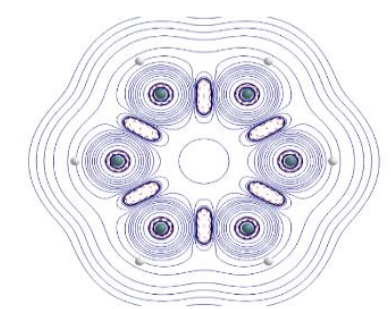

d)

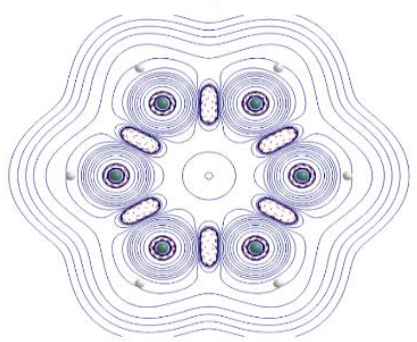

e)

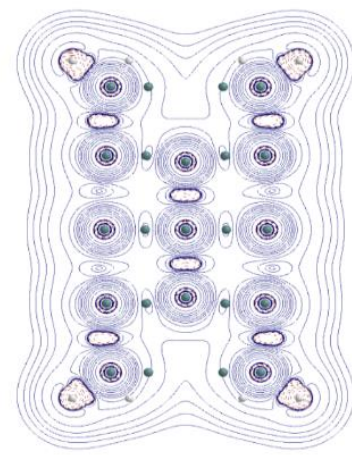

f)

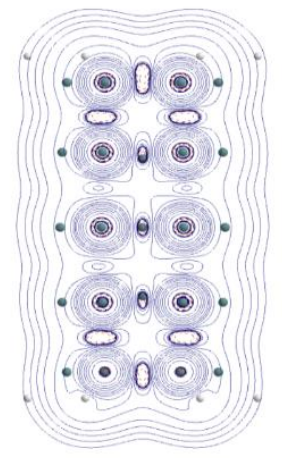

g)

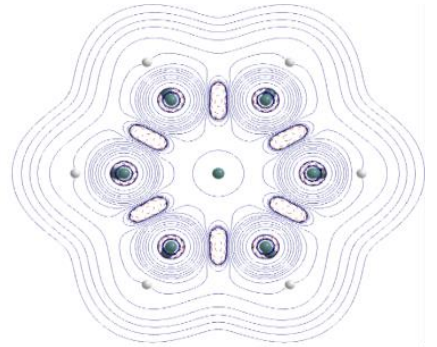

h)

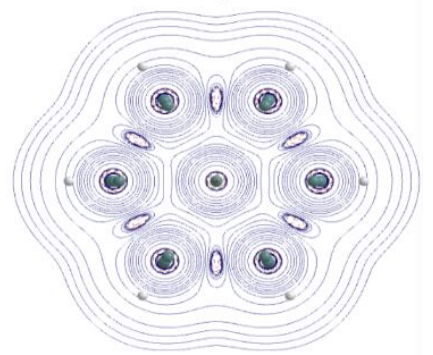

i)

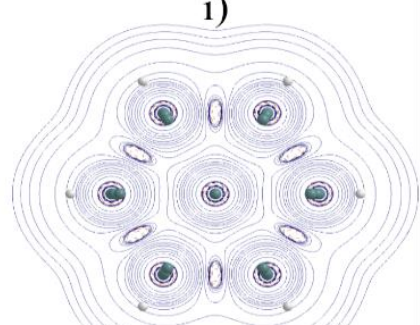

j)

Figure 3. The Laplacian contour map for the $\mathrm{Ge}_{30} \mathrm{H}_{12}$ in the plane: a) (Ge1Ge4H34), b) (Ge8Ge9Ge15), c) (Ge1Ge2Ge3), d) (Ge7Ge8Ge9), and e) (Ge13Ge14G15); and for $\mathrm{Ge}_{33} \mathrm{H}_{12}$ in the plane: f) (Ge3Ge23H42), g) (Ge1Ge2Ge24), h) (Ge1Ge2Ge3), i) (Ge7Ge8Ge9), and j) (Ge26Ge27G28). Dashed lines indicate charge concentrations, solid lines indicate charge depletions. Labels of atoms shown in Figure 1. 


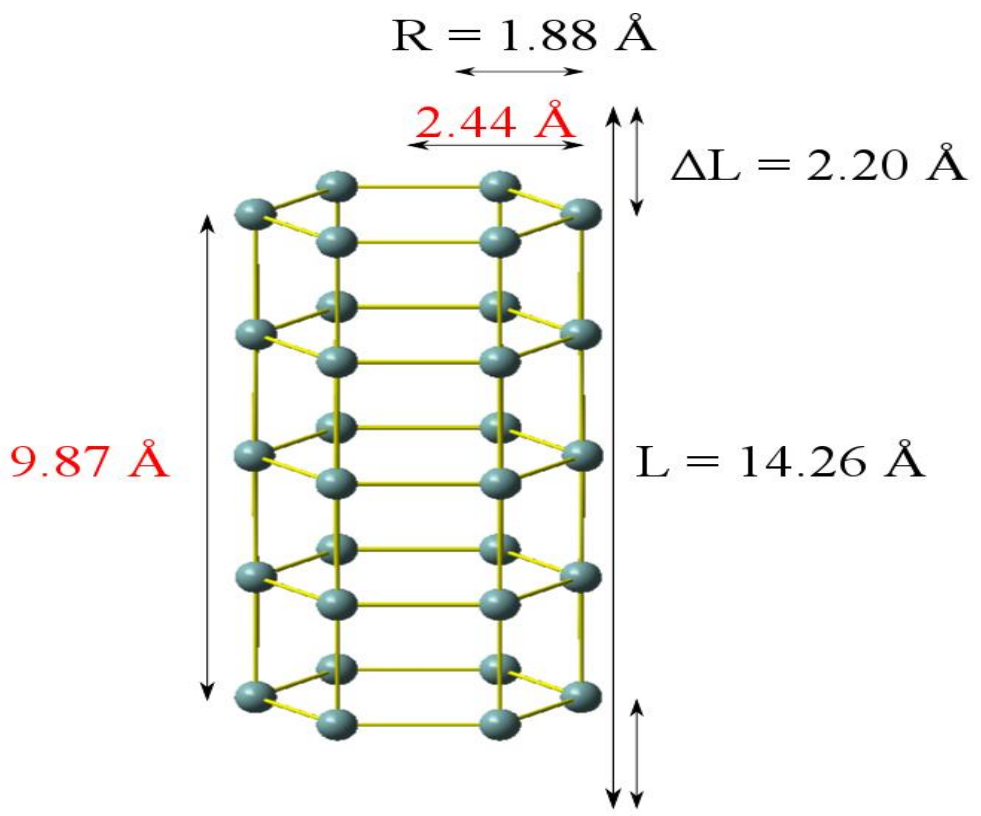

$\mathrm{Ge}_{30}$ skeleton

a)

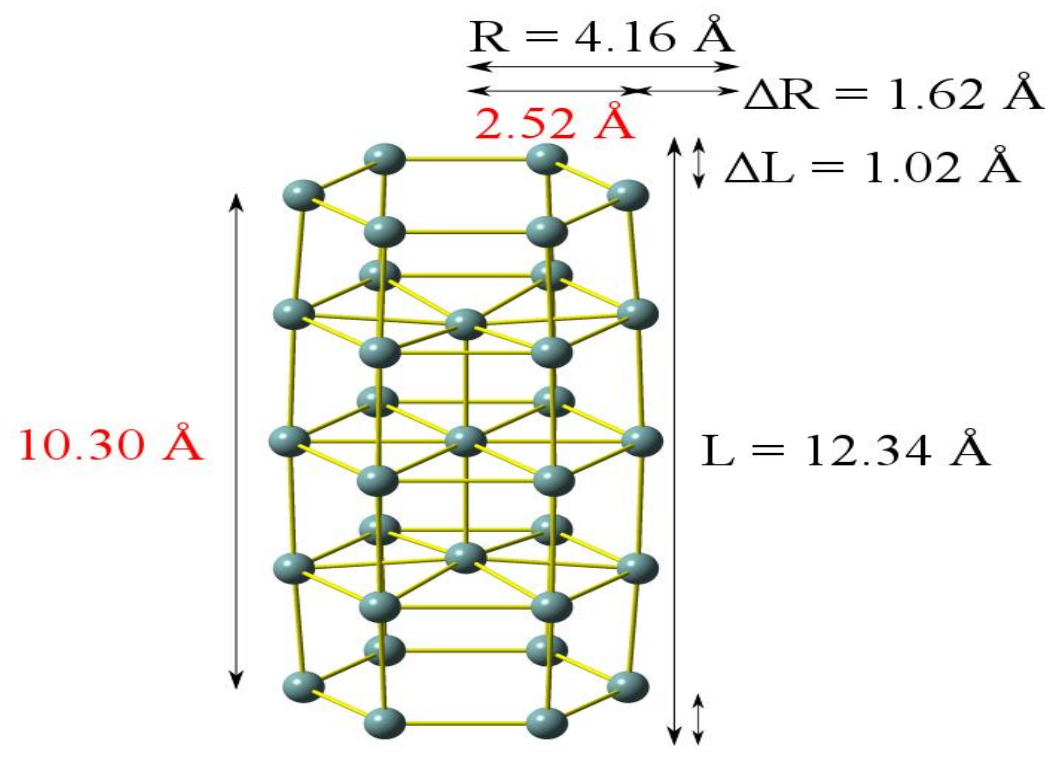

$\mathrm{Ge}_{33}$ skeleton

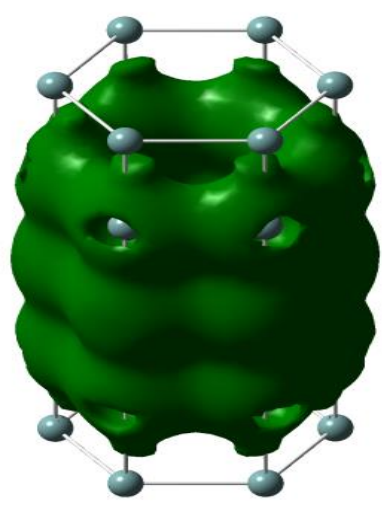

$\mathrm{HOMO}-40$

b)

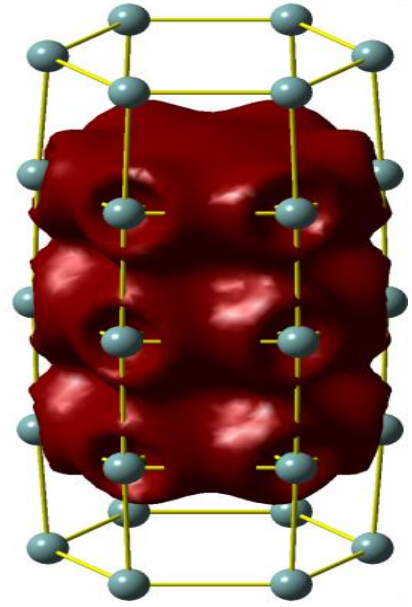

$\mathrm{HOMO}-45$

c)

d)

Figure 4. a) The $\mathrm{Ge}_{30}$ skeleton of the $\mathrm{Ge}_{30} \mathrm{H}_{12}$. The height of the skeleton is $9.87 \AA$, while the height of the hollow cylinder in which the electron is moving in is $14.26 \AA$. The extended length is $2.20 \AA$. The average radius of the skeleton is $2.44 \AA$ and the radius active of the hollow cylinder is $1.88 \AA$. b) The HOMO - 40 of the skeleton $\mathrm{Ge}_{30}$. c) The skeleton $\mathrm{Ge}_{33}$ of the $\mathrm{Ge}_{33} \mathrm{H}_{12}$. The height of the skeleton is $10.30 \AA$ while the height of the cylinder in which electron is moving in is $12.34 \AA$. The extended length is $1.02 \AA$. The average radius of the skeleton is $2.52 \AA$ and the radius of the cylinder is $4.16 \AA$. d) The HOMO -45 of the skeleton $\mathrm{Ge}_{33}$. 


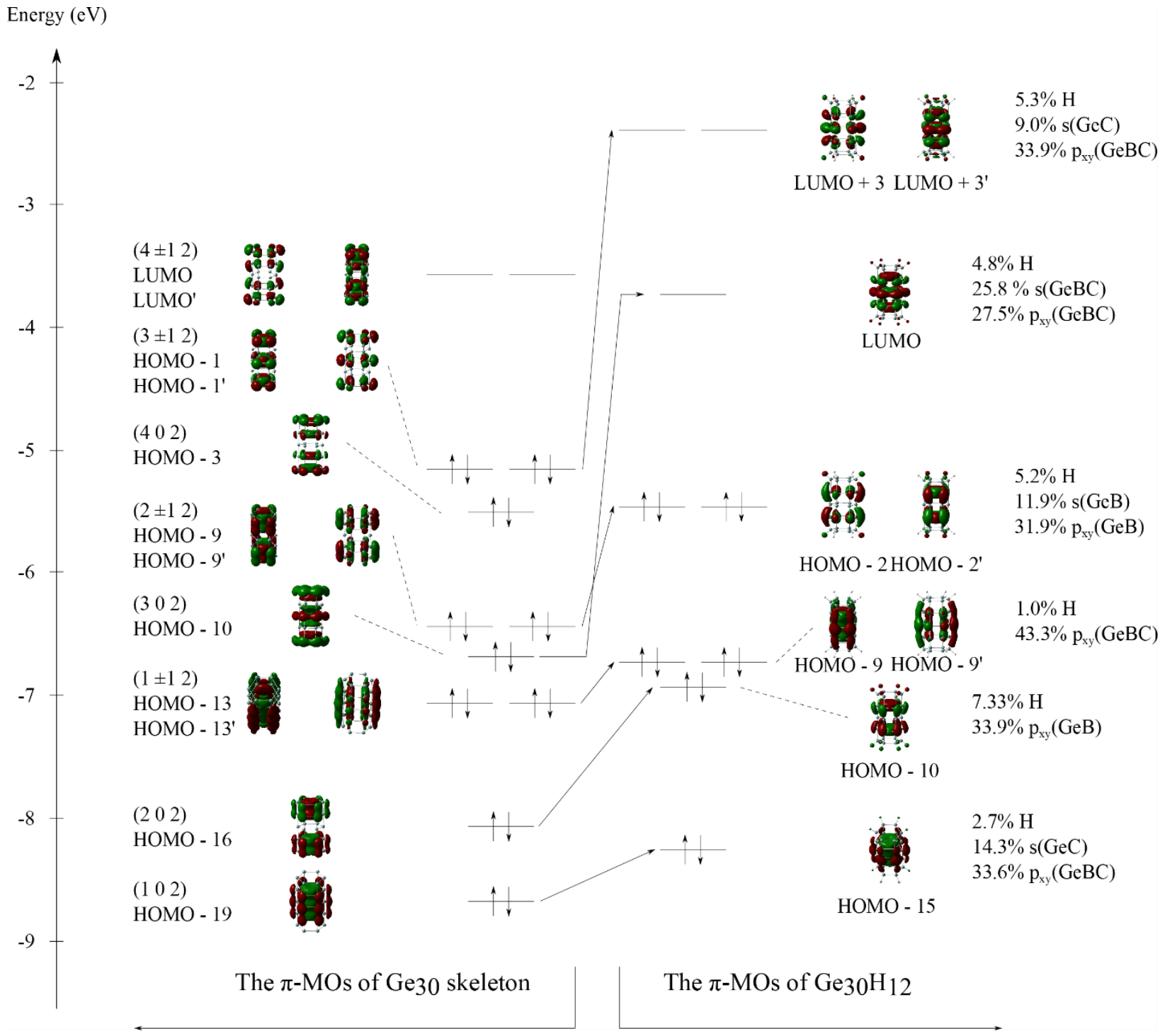

Figure 5. The formation of $\pi$-MOs set of $\mathrm{Ge}_{30} \mathrm{H}_{12}$ from the interaction between $\pi$-MOs set of skeleton $\mathrm{Ge}_{30}$ and 12 hydrogen atoms. 


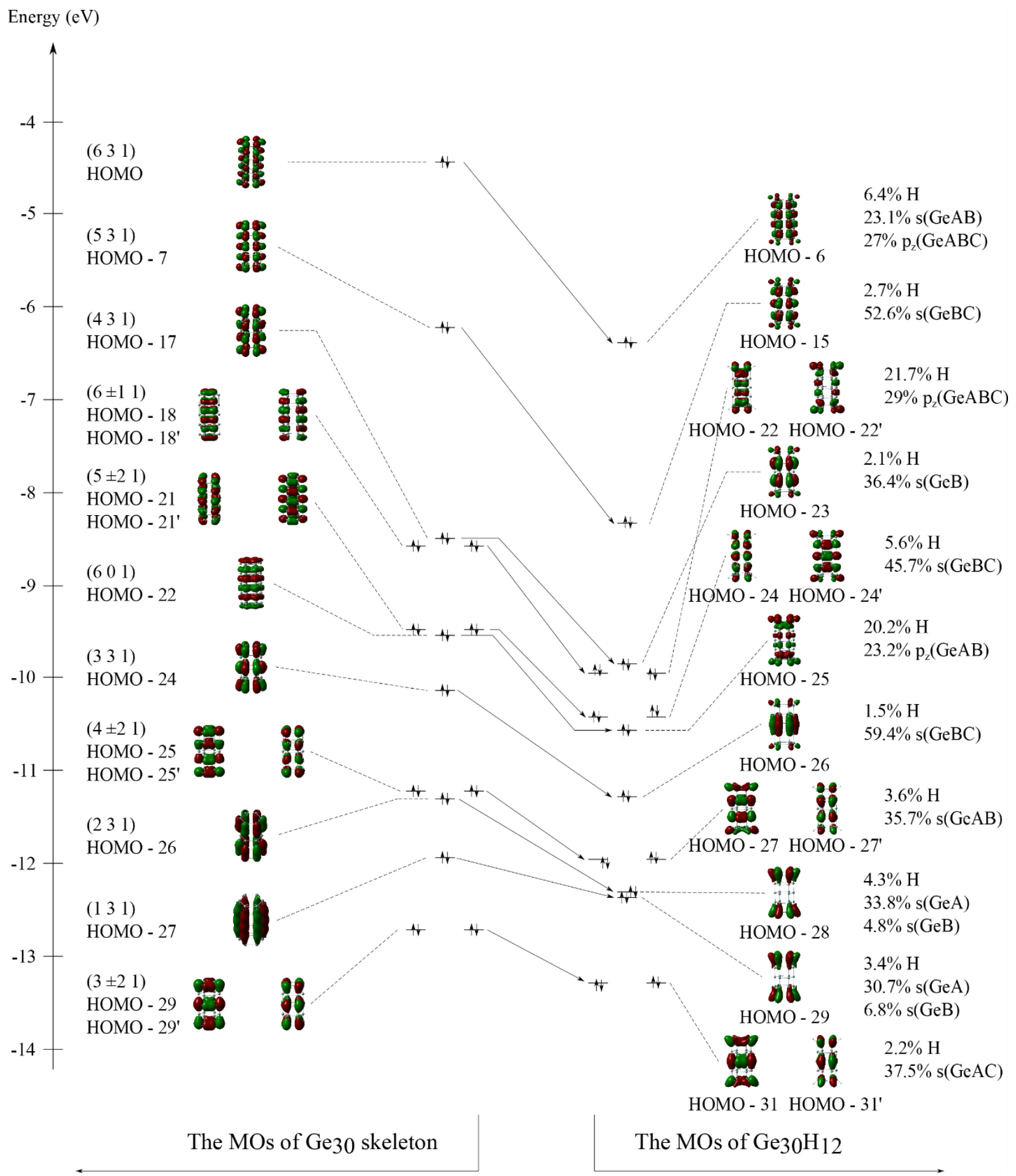

Figure 6. The formation of some $\sigma-\mathrm{MOs}$ of $\mathrm{Ge}_{30} \mathrm{H}_{12}$ from the interaction between $\sigma-\mathrm{MOs}$ of skeleton $\mathrm{Ge}_{30}$ and 12 hydrogen atoms which having the largest $|\Delta \mathrm{MO}|$ values. 


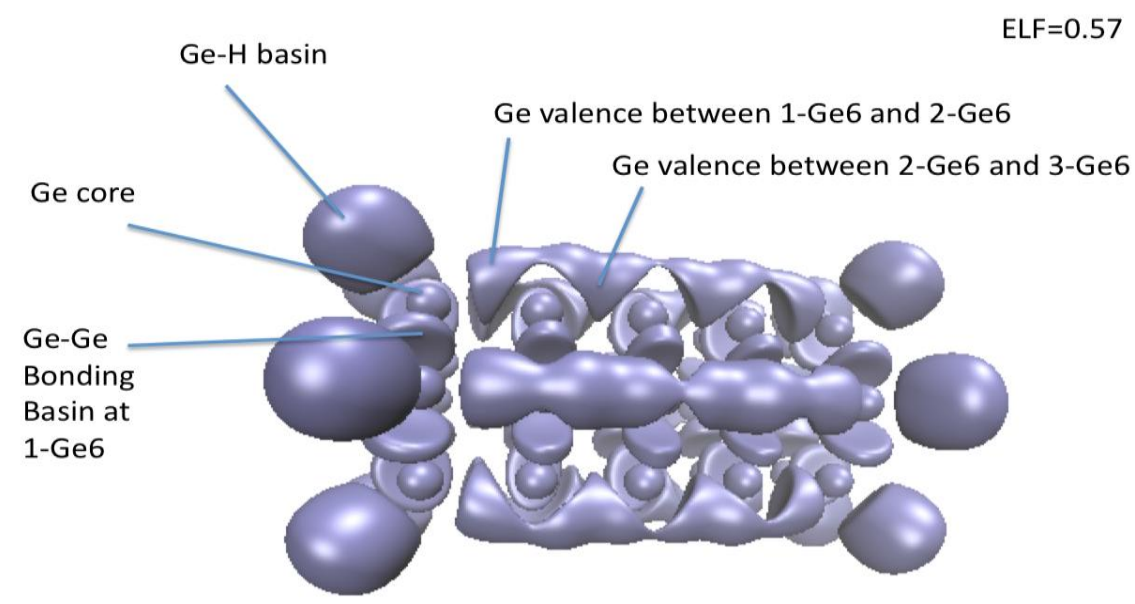

Figure 7. Electron localization function of $\mathrm{Ge}_{30} \mathrm{H}_{12}$ obtained at the B3LYP/6-31G(d) level of theory. Isosurface value of 0.57 . 


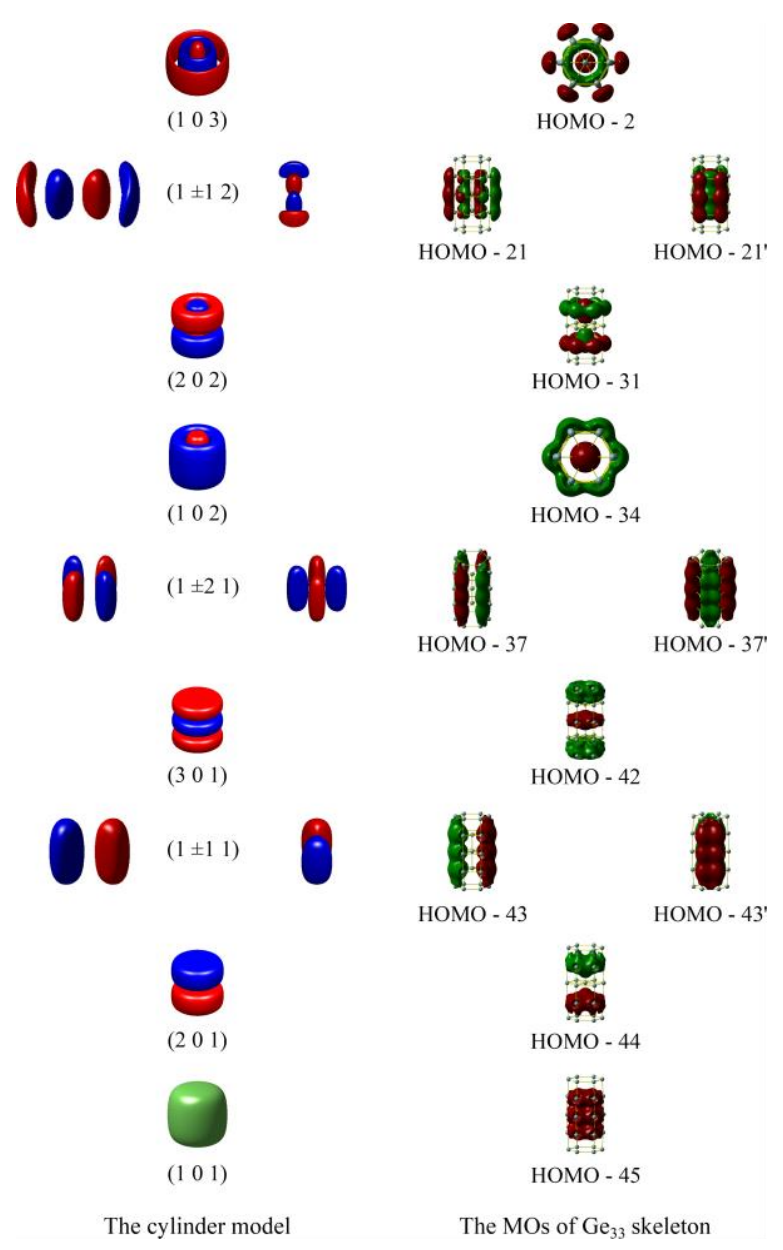

Figure 8. The comparison between the shape from cylinder model and the selected MOs from $\mathrm{Ge}_{33}$ skeleton. 


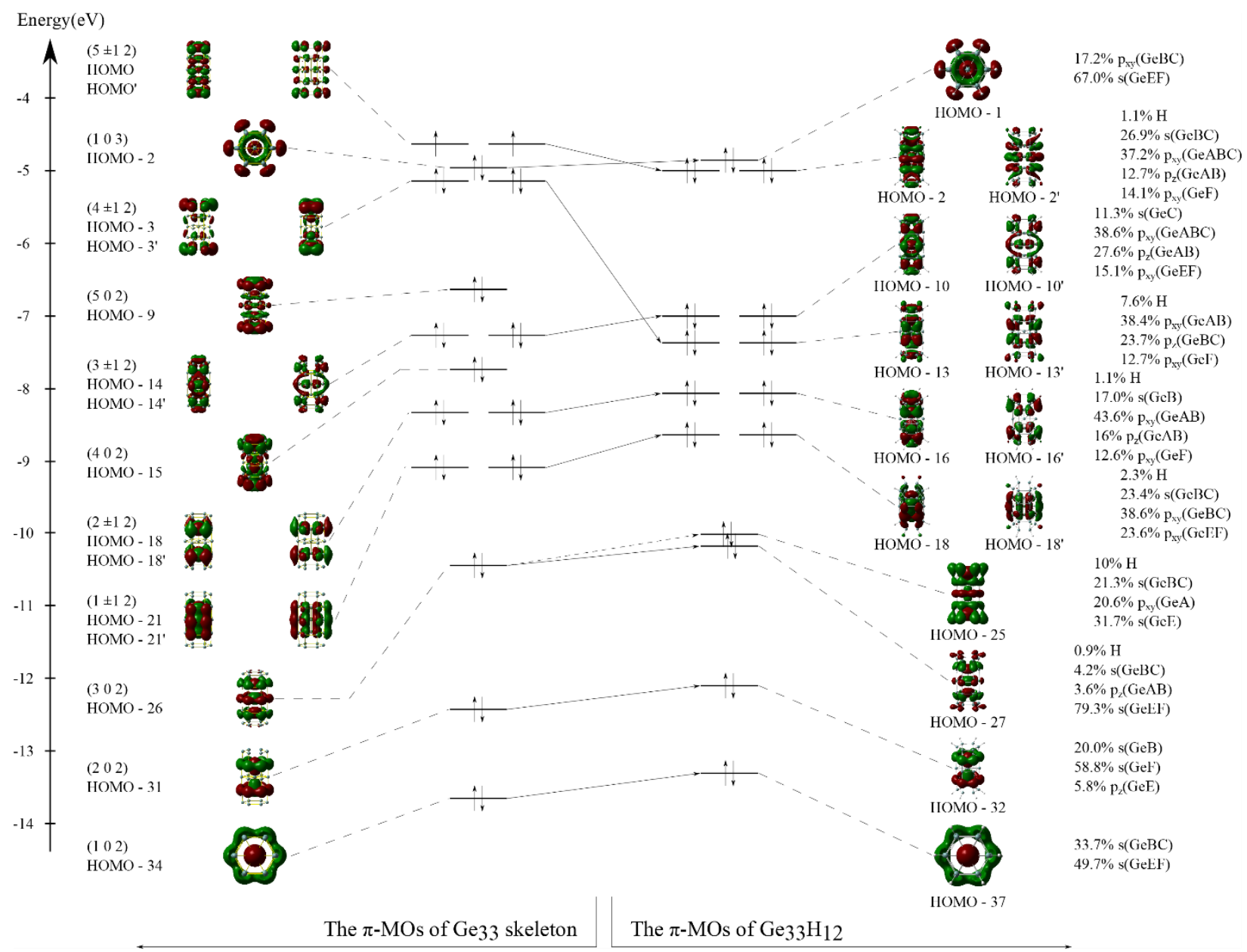

Figure 9. The formation of $\pi$-MOs set of $\mathrm{Ge}_{33} \mathrm{H}_{12}$ from the interaction between $\pi$-MOs set of skeleton $\mathrm{Ge}_{33}$ and 12 hydrogen atoms, $\mathrm{Ge}_{33}$ skeleton are calculated at single point after removing $12 \mathrm{H}$ (without optimization). 


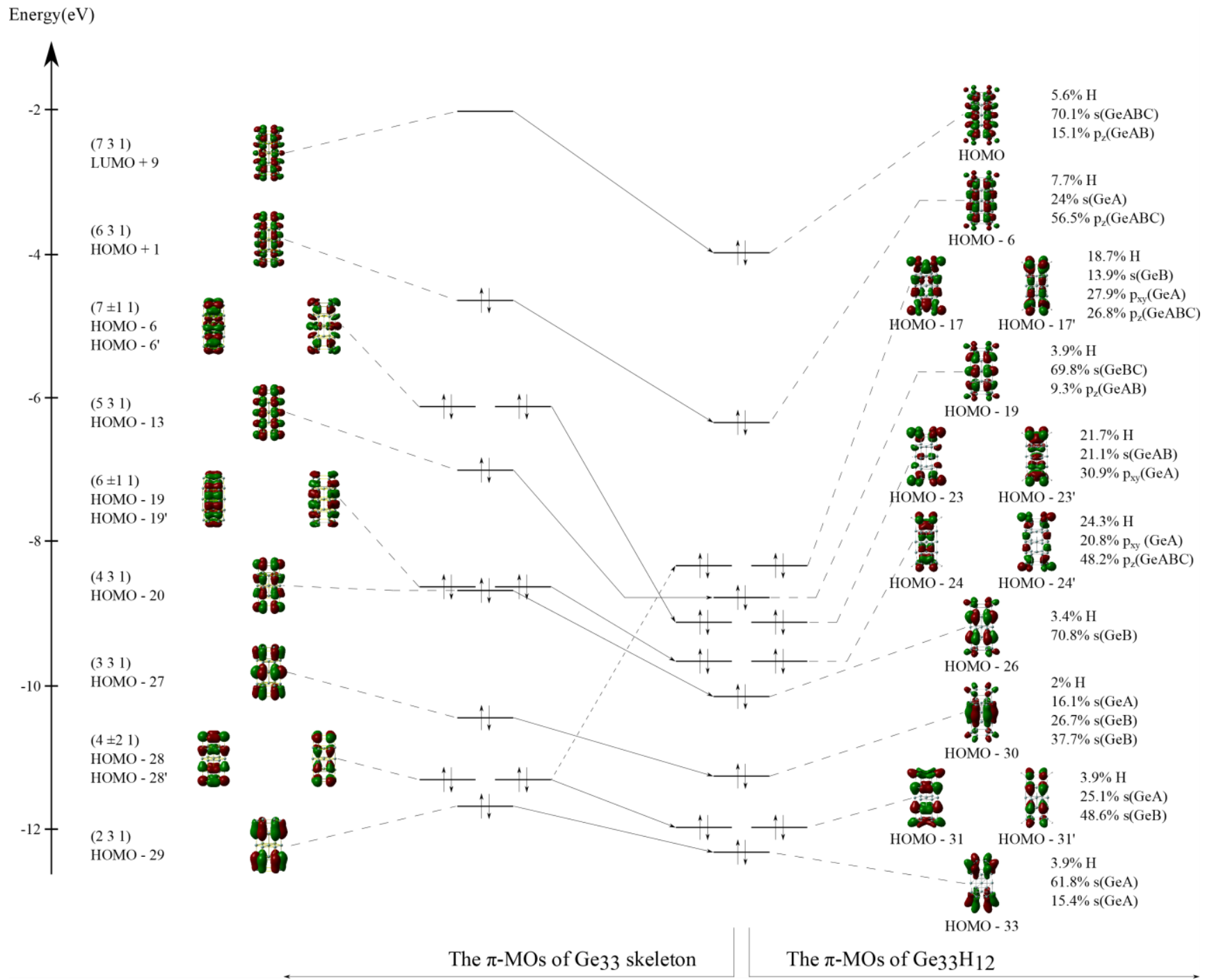

Figure 10. The formation of some $\sigma$-MOs of $\mathrm{Ge}_{33} \mathrm{H}_{12}$, which, from the interaction between $\sigma$ MOs of skeleton $\mathrm{Ge}_{33}$ and 12 hydrogen atoms. 


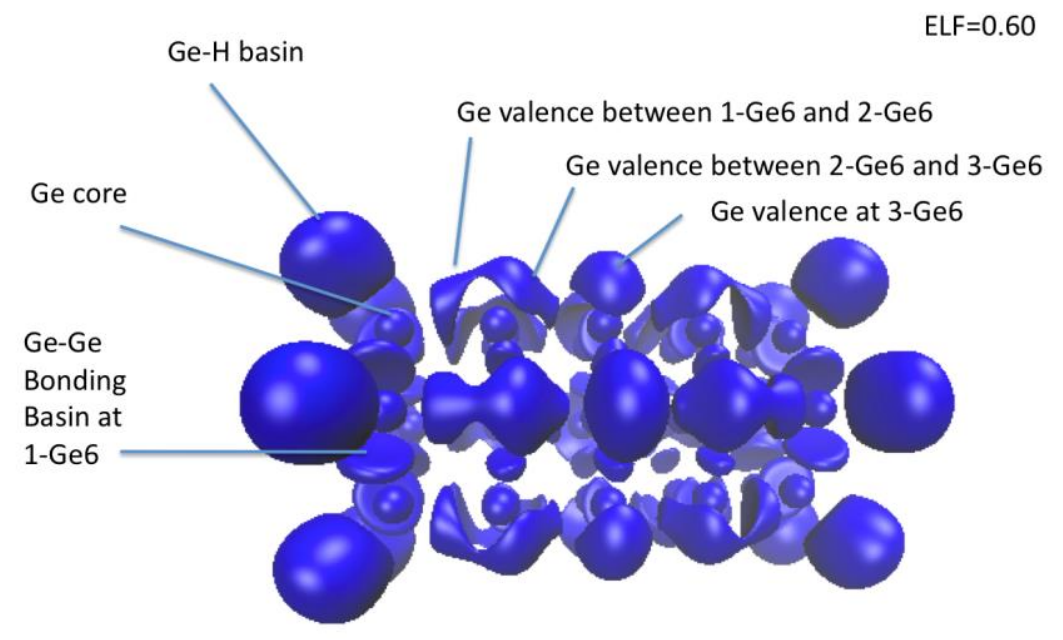

Figure 11. Electron localization function of $\mathrm{Ge}_{33} \mathrm{H}_{12}$ obtained at the B3LYP/6-31G(d) level of theory. Isosurface value of 0.60 . 


\section{References}

1. R. Gershoni-Poranne and A. Stanger, Magnetic criteria of aromaticity, Chem Soc Rev, 2015, 44, 6597-6615.

2. L. Zhao, R. Grande-Aztatzi, C. Foroutan-Nejad, J. M. Ugalde, G. Frenking, Aromaticity, the Hückel 4N + 2 Rule and Magnetic Current. ChemistrySelect 2017, 2, 863-870.

3. Z. Badri, C. Foroutan-Nejad, Unification of Ground-State Aromaticity Criteria-Structure, Electron Delocalization, and Energy-in Light of the Quantum Chemical Topology. Phys Chem Chem Phys 2016, 18, 11693-11699.

4. T. Janda, C. Foroutan-Nejad, Why Is Benzene Unique? Screening Magnetic Properties of $\mathrm{C}_{6} \mathrm{H}_{6}$ Isomers. ChemPhysChem 2018. doi:10.1002/cphc.201800364.

5. F. Feixas, E. Matito, J. Poater and M. Solà, On the performance of some aromaticity indices: A critical assessment using a test set, J Comput Chem, 2008, 29, 1543-1554.

6. H. Vach, L. V. Ivanova, Q. K. Timerghazin, F. Jardali and H.-L. T. Le, Metallic-like bonding in plasma-born silicon nanocrystals for nanoscale bandgap engineering, Nanoscale, 2016, 8, 18062-18069.

7. M. D. Peeks, T. D. Claridge and H. L. Anderson, Aromatic and antiaromatic ring currents in a molecular nanoring, Nature, 2017, 541, 200-203.

8. A. I. Boldyrev and L.-S. Wang, All-metal aromaticity and antiaromaticity, Chem Rev, 2005, 105, 3716-3757.

9. C. A. Tsipis, DFT study of "all-metal" aromatic compounds, Coordinate Chem Rev, 2005, 249, 2740-2762.

10. D. Y. Zubarev, B. B. Averkiev, H.-J. Zhai, L.-S. Wang and A. I. Boldyrev, Aromaticity and antiaromaticity in transition-metal systems, Phys Chem Chem Phys, 2008, 10, 257-267.

11. F. Feixas, E. Matito, J. Poater and M. Solà, Metalloaromaticity, Wires Comput Mol Sci, 2013, 3, 105-122.

12. J. Poater, M. Duran, M. Solà and B. Silvi, Theoretical evaluation of electron delocalization in aromatic molecules by means of atoms in molecules (AIM) and electron localization function (ELF) topological approaches, Chem Rev, 2005, 105, 3911-3947.

13. F. Feixas, E. Matito, J. Poater and M. Solà, Quantifying aromaticity with electron delocalisation measures, Chem Soc Rev, 2015, 44, 6434-6451.

14. A. R. Katritzky, P. Barczynski, G. Musumarra, D. Pisano and M. Szafran, Aromaticity as a quantitative concept. 1. A statistical demonstration of the orthogonality of classical and magnetic aromaticity in five-and six-membered heterocycles, J Am Chem Soc, 1989, 111, 7-15.

15. M. Giambiagi, M. S. de Giambiagi, C. D. dos Santos Silva and A. P. de Figueiredo, Multicenter bond indices as a measure of aromaticity, Phys Chem Chem Phys, 2000, 2, 3381-3392.

16. A. Stanger, What is... aromaticity: a critique of the concept of aromaticity — can it really be defined?, Chem Commun, 2009, 1939-1947.

17. J. Poater, X. Fradera, M. Duran and M. Solà, The delocalization index as an electronic aromaticity criterion: application to a series of planar polycyclic aromatic hydrocarbons, Chem Eur J, 2003, 9, 400406.

18. M. Solà, Why aromaticity is a suspicious concept? Why?, Front. Chem., 2017, 5, 22.

19. H. Vach, Symmetric and irregular aromatic silicon nanoclusters, Chem Phys Lett, 2014, 614, 199203.

20. R. J. Berger, H. S. Rzepa, D. Scheschkewitz, Ring Currents in the Dismutational Aromatic $\mathrm{Si}_{6} \mathrm{R}_{6}$. Angew. Chem. Int. Edit. 2010, 49, 10006-10009.

21. K. Abersfelder, A. J. White, H. S. Rzepa and D. Scheschkewitz, A tricyclic aromatic isomer of hexasilabenzene, Science, 2010, 327, 564-566. 
22. V. Svrcek, S. Cook, S. Kazaoui and M. Kondo, Silicon Nanocrystals and Semiconducting SingleWalled Carbon Nanotubes Applied to Photovoltaic Cells. J Phys Chem Lett 2011, 2, 1646-1650.

23. X. L. Wu, Y. G. Guo and L. J. Wan, Rational Design of Anode Materials Based on Group IVA Elements (Si, Ge, and Sn) for Lithium-Ion Batteries. Chem. Asian J. 2013, 8, 1948-1958.

24. K.-Y. Cheng, R. Anthony, U. R. Kortshagen and R. J. Holmes,Cheng, K.-Y.; Anthony, R.; Kortshagen, U. R.; Holmes, R. J., High-Efficiency Silicon Nanocrystal Light-Emitting Devices. Nano Lett 2011, 11, 1952-1956.

25. C. Bonafos, M. Carrada, G. Benassayag, S. Schamm-Chardon, J. Groenen, V. Paillard, B. Pecassou, A. Claverie, P. Dimitrakis and E. Kapetanakis, Si and Ge Nanocrystals for Future Memory Devices. Mater Sci Semicond Process. 2012, 15, 615-626.

26. X. Li, Z. Yang, Y. Fu, L. Qiao, D. Li, H. Yue and D. He, Germanium Anode with Excellent Lithium Storage Performance in a Germanium/Lithium-Cobalt Oxide Lithium-Ion Battery. ACS Nano 2015, 9 , $1858-1867$.

27. H. Vach, Ultrastable silicon nanocrystals due to electron delocalization, Nano Lett, 2011, 11, 54775481.

28. M. V. Gordeychuk, K. P. Katin, K. S. Grishakov and M. M. Maslov, Silicon buckyballs versus prismanes: Influence of spatial confinement on the structural properties and optical spectra of the $\mathrm{Si}_{18} \mathrm{H}_{12}$ and $\mathrm{Si}_{19} \mathrm{H}_{12}$ clusters, Int J Quantum Chem, 2018, e25609.

29. S. Manzetti, T. Lu, H. Behzadi, M. D. Estrafili, H.-L. T. Le and H. Vach, Intriguing properties of unusual silicon nanocrystals, $R S C A d v, 2015, \mathbf{5}, 78192-78208$.

30. H. Behzadi, Aromatic-like behavior of germanium nanocrystals, Rsc Adv, 2016, 6, 47434-47442.

31. R. King, I. Silaghi-Dumitrescu and M. Uţă, Beyond the Wade-Mingos Rules in Bare 10-and 12Vertex Germanium Clusters: Transition States for Symmetry Breaking Processes, J Chem Theory Comput, 2008, 4, 209-215.

32. L. V. Duong, H. T. Pham, N. M. Tam and M. T. Nguyen, A particle on a hollow cylinder: the triple ring tubular cluster $\mathrm{B}_{27}{ }^{+}$, Phys Chem Chem Phys, 2014, 16, 19470-19478.

33. H. T. Pham, L. V. Duong and M. T. Nguyen, Electronic structure and chemical bonding in the double ring tubular boron clusters, J Phys Chem C, 2014, 118, 24181-24187.

34. J. Gravesen, M. Willatzen and L. Lew Yan Voon, Schrödinger problems for surfaces of revolution - the finite cylinder as a test example, J Math Phys, 2005, 46, 012107.

35. A. D. Becke, Density-functional thermochemistry. III. The role of exact exchange, J Chem Phys, 1993, 98, 5648-5652.

36. C. Lee, W. Yang and R. G. Parr, Development of the Colle-Salvetti correlation-energy formula into a functional of the electron density, Phys Rev B, 1988, 37, 785.

37. P. Stephens, F. Devlin, C. Chabalowski and M. J. Frisch, Ab initio calculation of vibrational absorption and circular dichroism spectra using density functional force fields, J Phys Chem, 1994, 98, $11623-11627$.

38. M. Frisch, G. Trucks, H. B. Schlegel, G. Scuseria, M. Robb, J. Cheeseman, G. Scalmani, V. Barone, B. Mennucci and G. Petersson, Gaussian 09, revision A.02, Gaussian, Inc, Wallingford, CT, 2009.

39. G. Merino, A. Vela and T. Heine, Description of electron delocalization via the analysis of molecular fields, Chem Rev, 2005, 105, 3812-3841.

40. J. Poater, X. Fradera, M. Duran and M. Solà, An insight into the local aromaticities of polycyclic aromatic hydrocarbons and fullerenes, Chem Eur J, 2003, 9, 1113-1122.

41. X. Fradera, M. A. Austen and R. F. Bader, The Lewis model and beyond, J Phys Chem A, 1999, 103, 304-314.

42. X. Fradera, J. Poater, S. Simon, M. Duran and M. Solà, Electron-pairing analysis from localization and delocalization indices in the framework of the atoms-in-molecules theory, Theor Chem Acc, 2002, 108, 214-224.

43. R. Bader. Atoms in Molecules: A Quantum Theory: Oxford Univ. Press. Oxford; 1990.

44. R. F. Bader, Atoms in molecules, Acc Chem Res, 1985, 18, 9-15. 
45. R. F. Bader, A quantum theory of molecular structure and its applications, Chem Rev, 1991, 91, 893-928.

46. I. Mayer, Charge, bond order and valence in the AB initio SCF theory, Chem Phys Lett, 1983, 97, 270-274.

47. J. Cioslowski, E. Matito and M. Solà, Properties of aromaticity indices based on the one-electron density matrix, J Phys Chem A, 2007, 111, 6521-6525.

48. I. Gutman, M. Milun and N. Trinajstic, Graph theory and molecular orbitals. 19. Nonparametric resonance energies of arbitrary conjugated systems, J Am Chem Soc, 1977, 99, 1692-1704.

49. E. Matito, F. Feixas and M. Solà, Electron delocalization and aromaticity measures within the Hückel molecular orbital method, J Mol Struct Theochem, 2007, 811, 3-11.

50. P. Bultinck, R. Ponec and S. Van Damme, Multicenter bond indices as a new measure of aromaticity in polycyclic aromatic hydrocarbons, J Phys Org Chem, 2005, 18, 706-718.

51. E. Matito, An electronic aromaticity index for large rings, Phys Chem Chem Phys, 2016, 18, 1183911846.

52. F. W. Biegler-könig, R. F. Bader and T. H. Tang, Calculation of the average properties of atoms in molecules. II, J Comput Chem, 1982, 3, 317-328.

53. E. Matito. ESI-3D: electron sharing indices program for 3D molecular space partitioning. Institute of Computational chemistry and Catalysis (IQCC), University of Girona, Catalonia, Spain. University of Girona: Catalonia, Spain; 2006.

54. E. Matito, M. Duran and M. Solà, The aromatic fluctuation index (FLU): a new aromaticity index based on electron delocalization, J Chem Phys, 2005, 122, 014109; erratum, íbid., 2006, 125, 059901.

55. E. Matito, M. Solà, P. Salvador and M. Duran, Electron sharing indexes at the correlated level. Application to aromaticity calculations, Faraday Discuss, 2007, 135, 325-345.

56. J. Poater, M. Solà, M. Duran and X. Fradera, The calculation of electron localization and delocalization indices at the Hartree-Fock, density functional and post-Hartree-Fock levels of theory, Theor Chem Acc, 2002, 107, 362-371.

57. S. Noury, X. Krokidis, F. Fuster and B. Silvi, Computational tools for the electron localization function topological analysis, Computers \& Chemistry, 1999, 23, 597-604.

58. A. Savin, R. Nesper, S. Wengert and T. F. Fässler, ELF: The electron localization function, Angew Chem Int Edit, 1997, 36, 1808-1832.

59. S. Noury, F. Colonna, A. Savin and B. Silvi, Analysis of the delocalization in the topological theory of chemical bond, J Mol Struct, 1998, 450, 59-68.

60. A. D. Becke and K. E. Edgecombe, A simple measure of electron localization in atomic and molecular systems, J Chem Phys, 1990, 92, 5397-5403.

61. A. Savin, B. Silvi and F. Colonna, Topological analysis of the electron localization function applied to delocalized bonds, Can J Chem, 1996, 74, 1088-1096.

62. J. Santos, W. Tiznado, R. Contreras and P. Fuentealba, Sigma-pi separation of the electron localization function and aromaticity, J Chem Phys, 2004, 120, 1670-1673.

63. L. Van Duong and M. T. Nguyen, Electronic structure of the boron fullerene $B_{14}$ and its silicon derivatives $\mathrm{B}_{13} \mathrm{Si}^{+}, \mathrm{B}_{13} \mathrm{Si}^{-}$and $\mathrm{B}_{12} \mathrm{Si}_{2}$ : a rationalization using a cylinder model, Phys Chem Chem Phys, 2016, 18, 17619-17626.

64. E. Miliordos, Hückel versus Möbius aromaticity: The particle in a cylinder versus a Möbius strip, Phys Rev A, 2010, 82, 062118.

65. T. Papp, L. Kollár and T. Kégl, Theoretical insights into the nature of Pt-Sn bond: Reevaluating the bonding/back-bonding properties of trichlorostannate with comparison to the cyano ligand, $J$ Comput Chem, 2017, 38, 1712-1726.

66. R. F. Bader and H. Essén, The characterization of atomic interactions, J Chem Phys, 1984, 80, 1943-1960.

67. I. Mayer, Bond order and valence: Relations to Mulliken's population analysis, Int J Quantum Chem, 1984, 26, 151-154. 
68. H. T. Pham, T.-T. Phan, N. M. Tam, L. V. Duong, M. P. Pham-Ho and M. T. Nguyen, $\mathrm{Mn}_{2} @ \mathrm{Si}_{15}$ : the smallest triple ring tubular silicon cluster, Phys Chem Chem Phys, 2015, 17, 17566-17570.

69. M. Mantina, A. C. Chamberlin, R. Valero, C. J. Cramer and D. G. Truhlar, Consistent van der Waals radii for the whole main group, J Phys Chem A, 2009, 113, 5806-5812.

70. L. Van Duong and M. T. Nguyen, On the role of different types of electron in double ring tubular clusters, Chem Phys Lett, 2017, 685, 377-384. 